\title{
Crystalline versus Amorphous Donor-Acceptor Blends: Influence of Layer Morphology on the Charge-Transfer Density of States
}

\author{
Theresa Linderl, ${ }^{1}$ Thomas Zechel, ${ }^{1}$ Alexander Hofmann, ${ }^{1}$ Tomoya Sato, ${ }^{2}$ Kohei Shimizu $\odot,{ }^{2}$ \\ Hisao Ishii, ${ }^{2}$ and Wolfgang Brütting $\oplus^{1, *}$ \\ ${ }^{1}$ Institute of Physics, University of Augsburg, 86135 Augsburg, Germany \\ ${ }^{2}$ Center for Frontier Science, Chiba University, Chiba 263-8522, Japan
}

(Received 4 September 2019; revised manuscript received 14 January 2020; accepted 24 January 2020; published 24 February 2020)

\begin{abstract}
Organic small molecule solar cells are used as a test bed to investigate the influence of film morphology on the density of charge-transfer (CT) states. CT states are considered as precursors for charge generation and their energy as the upper limit for the open-circuit voltage in organic donor-acceptor solar cells. In this study the influence of morphology for two perylene donors [crystalline diindenoperylene (DIP) versus amorphous tetraphenyldibenzoperiflanthene (DBP)] with almost identical ionization energy is investigated. As acceptor material, the fullerene $\mathrm{C}_{60}$ is used. By combining device measurements with optical and low-energy ultraviolet photoelectron spectroscopy, a comprehensive picture is obtained that describes how morphology and the connected density of states (DOS) affect device performance and the spectroscopic signature of CT states. Especially for the crystalline donor material DIP, strong exponential tail states reaching far into the gap are observed, which can be related to the presence of grain boundaries. A voltage-dependent filling of these states is identified as the origin of a blue shift of electroluminescence spectra with increasing applied voltage. Different approaches are compared to study the influence of static and dynamic disorder in the description of CT emission and absorption spectra of organic solar cells. Despite the fact that both donors yield almost identical CT energy (and, thus, the same open-circuit voltage) the Stokes shift between photocurrent and electroluminescence spectra and, concomitantly, the width of the CT DOS varies by more than a factor of 2 . We discuss this observation in terms of the donoracceptor reorganization energy as well as an additional line broadening by static disorder. Remarkably, the more crystalline donor DIP shows a significant deviation from a Marcus-type description, while this is not the case for the amorphous DBP. This highlights the importance of film morphology in organic solar cells.
\end{abstract}

DOI: 10.1103/PhysRevApplied.13.024061

\section{INTRODUCTION}

Organic photovoltaics (OPVs) have seen a tremendous increase in power conversion efficiency over the last three decades and are now reaching top efficiencies of more than $16 \%$ [1]. However, the difference between the measured open-circuit voltage $V_{\mathrm{OC}}$ and the energy gap of the light-absorbing materials $E_{g}$, the so-called bandgapvoltage offset, is considerably larger for OPV materials than for inorganic semiconductor or perovskite solar cells $[2,3]$. One reason is that charge generation in OPVs occurs through charge-transfer (CT) states, which consist of Coulombically bound electron-hole pairs at the donoracceptor (DA) interface. Accordingly, detailed knowledge of the CT density of states (DOS) and its occupation under different excitation conditions is crucial for understanding OPV functioning. Central parameters are the CT energy

\footnotetext{
*wolfgang.bruetting@physik.uni-augsburg.de
}

$E_{\mathrm{CT}}$, i.e., the energy difference between the lowest CT state and the ground state, as well as the reorganization energy $\lambda$ due to changes of nuclear coordinates in the CT complex and its environment, which form the basis for the description of CT spectra in the framework of Marcus theory [4-8]. Other authors have pointed to the role of static disorder, the film morphology as well as a potentially nonthermal site occupation [9-16]. Regardless of the details, it is generally accepted that the CT DOS can be described by a Gaussian distribution (or a superposition of several Gaussians if vibronic progressions of higherenergy modes or the coexistence of different electronic DA configurations come into play). However, deviations from this generic shape will have important consequences reflected, e.g., in state-filling effects under varying light intensities or applied bias conditions. In particular, this can lead to strongly voltage-dependent electroluminescence spectra as well as nonideal recombination kinetics resulting potentially in ambiguities about the CT energy and 
related parameters. Differences between optical and electrical excitation can complicate the analysis additionally.

Here, we directly demonstrate the strong influence morphology has on the CT DOS and show how filling of tail states affects the optical spectra. We use two molecular donor materials with almost identical ionization energy and combine them with the fullerene $\mathrm{C}_{60}$ as acceptor. Remarkably, however, one of the two perylene donors, diindenoperylene (DIP), shows crystalline film growth, while the other one, tetraphenyldibenzoperiflanthene (DBP), is amorphous. We combine morphological investigations with sensitive ultraviolet photoelectron spectroscopy, as well as spectral electroluminescence and photocurrent measurements to study the consequences of different film morphologies for the CT DOS.

\section{METHODS}

Sample preparation: All samples are fabricated on commercially available indium-tin-oxide- (ITO) coated glass substrates (purchased from Thin Film Devices, Inc., Anaheim, CA; sheet resistance approximately equal to $20 \Omega$ /square). Prior to the evaporation of the active organic layers, a $30-\mathrm{nm}$-thick layer of poly $(3,4-$ ethylenedioxythiophene):poly(styrenesulfonate) (PEDOT: PSS; purchased from Heraeus Clevios $\mathrm{GmbH}$, Leverkusen, Germany as AI 4083 or HIL 1.3) is spin coated from an aqueous solution and annealed at $125^{\circ} \mathrm{C}$ for 30 min on a hot plate under ambient conditions. Subsequently, the active donor and acceptor layers as well as for the diodes a 5-nm layer of the exciton blocking material bathocuproine (BCP; purchased from Sigma Aldrich as sublimed grade and used without further purification) and $100 \mathrm{~nm}$ of aluminium are deposited using a standard thermal-evaporation procedure at base pressures of $10^{-7}$ mbar. The aluminium is evaporated through a shadow mask as a top electrode, resulting in an active area of $A=4 \mathrm{~mm}^{2}$. Thus the layer sequence for all devices is ITO/PEDOT:PSS/organic layers/BCP/Al. The active material diindenoperylene (DIP; purchased from S. Hirschmann, University of Stuttgart, Stuttgart, Germany) are purified twice by gradient sublimation and tetraphenyldibenzoperiflanthene (DBP) as well as fullerene $\mathrm{C}_{60}$ (both purchased from Lumtec, Taiwan) are used as received.

Ultraviolet photoelectron spectroscopy (UPS): UPS is performed using two different excitation light sources (UV and VIS), a Xe lamp and a $\mathrm{D}_{2}$ lamp for the energy ranges of $1.5-6.5$ and 3.6-8.0 eV, respectively. To eliminate stray light, a zero-dispersion double monochromator (Bunloukeiki M25GTM-DZ) is adapted to the light sources. The incident angle of the light beam is $55^{\circ}$ from the surface normal and emitted photoelectrons are detected at the surface normal direction with a $120-\mathrm{mm}$ hemispherical analyzer
(PSP Vacuum Technology RESOLVE120). All measurements are performed under a sample bias of $-10 \mathrm{~V}$ and the total energy resolution of the setup is $0.23 \mathrm{eV}$. The samples are prepared in a separate preparation chamber and are subsequently transferred into the measurement chamber without breaking vacuum. Both chambers have a base preassure below $5 \times 10^{-7} \mathrm{~Pa}$.

Morphological characterization: Morphological characterization is performed by an atomic force microscope (AFM, Thermo Microscopes Autoprobe CP-Research) in tapping mode.

Electroluminescence (EL) measurements: EL measurements are carried out by using a liquid-nitrogen-cooled CCD camera (PyLoN:100BR_eXcelon, Princton Instruments) coupled with a spectrometer (SP2300i, Princton Instruments) with a spectral sensitivity in the wavelength range of approximately $300-1050 \mathrm{~nm}$. The measurements are performed under a dc voltage drive from a Keithley source meter. The samples were transfered into a liquidhelium-cooled cryostat (Cryovac) with an inert gas atmosphere (approximately $300 \mathrm{mbar} \mathrm{He}$ ) without air exposure.

Incident photon to current efficiency (IPCE): IPCE measurements are performed using a halogen lamp together with a monochromator (Omni- $\lambda 300$, Zolix Instruments Co., Ltd.), which allows wavelength-dependent measurements in the range from 350 to $1100 \mathrm{~nm}$ in steps of $1 \mathrm{~nm}$. The current signal of the solar cell is measured using a lock-in amplifier (Stanford Research Systems SR830) and compared to the signal of a Si-reference cell (Thorlabs, FDS 100) that is measured by a Keithley 237 SMU.

\section{RESULTS}

\section{A. Structure and morphology}

The structure and morphology of thin films of the two donor materials - DIP and, to a lesser extent, DBP as well - in combination with the fullerene $\mathrm{C}_{60}$ as acceptor have already been the subject of several investigations [17-19]. Thus, we only summarize the key findings here and refer to the literature for further details. For comparison with electrical device characteristics, the donorfullerene blends are fabricated on ITO-glass substrates covered with the hole-injection layer PEDOT:PSS. Prior to codeposition of the 50-nm-thick D:A (1:1) blend a thin neat donor layer (ca. $5 \mathrm{~nm}$ thickness) is deposited. Both of the layers are grown at an elevated substrate temperature of about $100^{\circ} \mathrm{C}$.

Although the chemical and electronic structure of the two donor molecules is quite similar (see Fig. 1, top), thin films show significant differences concerning morphology, molecular orientation, absorption, and transport properties [19,20]. While DIP is a flat molecule consisting of a perylene core with two indeno end groups, DBP has a more extended core with additional benzene rings on each end and, most importantly, four additional rotatable phenyl 


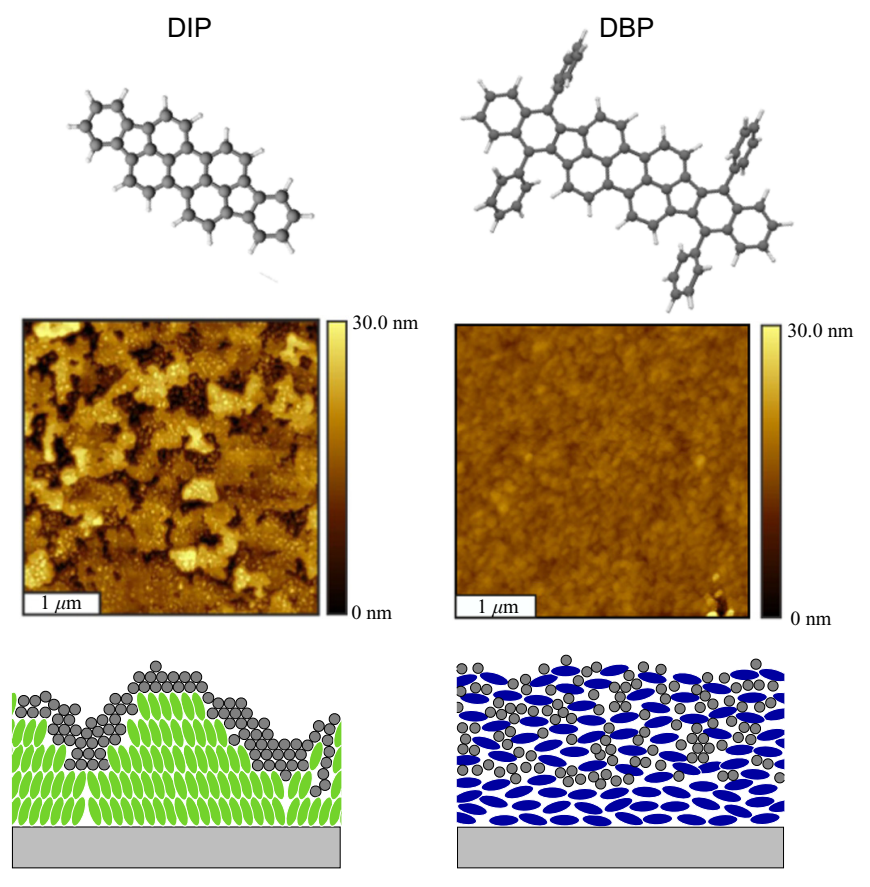

FIG. 1. Top: molecular structures of the donor materials DIP and DBP; center: AFM images of 1:1 mixtures of both donors with the fullerene $\mathrm{C}_{60}$ as acceptor; bottom: sketch of the layer morphology for both cases.

groups at the perylene core such that the molecule is no longer flat but rather bulky (compare the analogous case of tetracene and rubrene). Nevertheless, the similarity in their molecular cores leads to almost identical ionization energies of about $5.4-5.5 \mathrm{eV}[7,21,22]$, and consequently very similar DA gaps to the fullerene $\mathrm{C}_{60}$ leading to basically the same open-circuit voltage $V_{\mathrm{OC}}$ of ca. $0.9 \mathrm{~V}$ in solar cells [19] (see also Sec. I and II of the Supplemental Material [23]).

However, the small differences in their molecular structure, especially the four rotatable phenyl rings, induce fundamental differences concerning film growth. For DIP evaporated on various kinds of substrates highly crystalline films with almost vertically standing molecules are reported [18,24]. Domain sizes in these polycrystalline films can reach almost up to the micro-meter scale for heated substrates. By contrast, DBP does not exhibit measurable crystallinity regardless of growth temperature and the used substrate [25]. Still the films are not perfectly amorphous but show a preferentially lying orientation of the molecules, which is also confirmed by the huge difference in optical absorption of both materials (see Fig. SI1 of the Supplemental Material [23]). Thus, from an optical point of view, DBP is more favorable for solar-cell applications, which is supported by the corresponding device data (see Fig. SI2 and Table SI-I of the Supplemental Material [23]).

When coevaporated with the fullerene $\mathrm{C}_{60}$ to form a so-called bulk heterojunction, both donors keep their differences in film growth. DIP: $\mathrm{C}_{60}$ mixtures show pronounced phase separation with a noticeable reduction in domain sizes as compared to the neat DIP films at identical growth conditions [18]. However, even these bulk heterojunctions are highly crystalline such that charge transfer between donor and acceptor can only occur at grain boundaries between DIP and the fullerene. DBP and $\mathrm{C}_{60}$, on the other hand, exhibit intermixing on a molecular level over a wide range of mixing ratios without indications for crystallinity or large-scale phase separation $[19,26]$. All these findings support largely different scenarios for the resulting film morphology as shown in Fig. 1. DBP: $\mathrm{C}_{60}$ mixtures form smooth amorphous, spatially homogenous films, while the DIP: $\mathrm{C}_{60}$ counterpart has highly crystalline neat $\mathrm{D}$ domains (and to a lesser extent also $\mathrm{A}$ ) with very little intermixing and higher roughness. It was shown by earlier x-ray-diffraction studies that the vertical DIP domain size typically is as big as the film thickness in such crystalline D:A blends $[17,18]$. This means that in a solar cell with a thin neat $\mathrm{C}_{60}$ layer evaporated on top of the blend, $\mathrm{C}_{60}$ molecules will be sitting predominantly on edge-onoriented DIP molecules; nevertheless, there are also DIP grain boundaries, where face-on-exposed DIP molecules are in contact to $\mathrm{C}_{60}$.

In the following, we study the consequences of these structural differences for the electronic properties - and, in particular, the density and spectral properties of intermolecular CT states at the DA interface.

\section{B. Photoelectron spectroscopy}

For a detailed understanding of CT states it is crucial to investigate the electronic structure of the material combination of interest. In particular, states that possibly exist within the bandgap region between the energy of the highest occupied molecular orbital (HOMO) and the lowest unoccupied molecular orbital (LUMO) might strongly influence device performance, not only by affecting $V_{\mathrm{OC}}$ via the quasi-Fermi-level positions, but also by acting as recombination centers and thus reducing the charge-carrier mobility and the short-circuit current $j_{\mathrm{SC}}$ of the device [2730]. To determine the DOS distribution of the HOMO region of the donor, including tail states that reach far into the bandgap region, low-energy ultraviolet photoemission spectroscopy (LE UPS) measurements on neat donor layers as well as on 1:1 mixtures of the donors with the acceptor $\mathrm{C}_{60}$ are performed. To keep the samples used for UPS measurements as close as possible to the structure of solar cells, the films are prepared on ITO substrates using the PEDOT:PSS derivative HIL 1.3 as the hole injection layer. UPS spectra are recorded on neat $25-\mathrm{nm}$-thick donor layers as well as on 50-nm-thick 1:1 mixtures of the donors with $\mathrm{C}_{60}$. Several spectra with excitation energies between 7.7 and $4.5 \mathrm{eV}$ are recorded and combined to one overall spectrum with a high dynamic range that provides insight 
into the distribution of occupied states far into the gap of the investigated sample. The resulting spectra are shown in Fig. 2 (see the Supplemental Material for more information on how the spectra are obtained from a series of measurements with varying excitation energy). Neat donor layers are compared to D:A mixtures of the respective donor with the acceptor $\mathrm{C}_{60}$.

As shown in Fig. SI4 and SI5 of the Supplemental Material [23], the HOMO regions of all spectra can be fitted with Gaussian functions. They exhibit similar peak

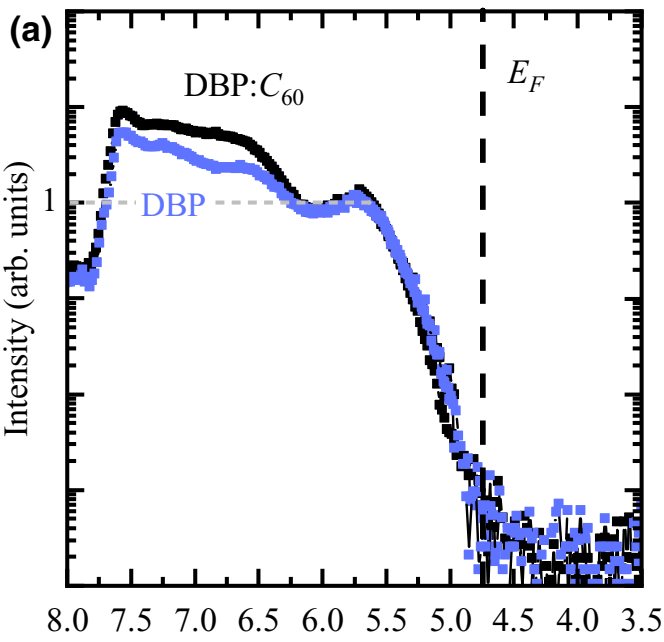

Binding energy relative to $\mathrm{VL}(\mathrm{eV})$

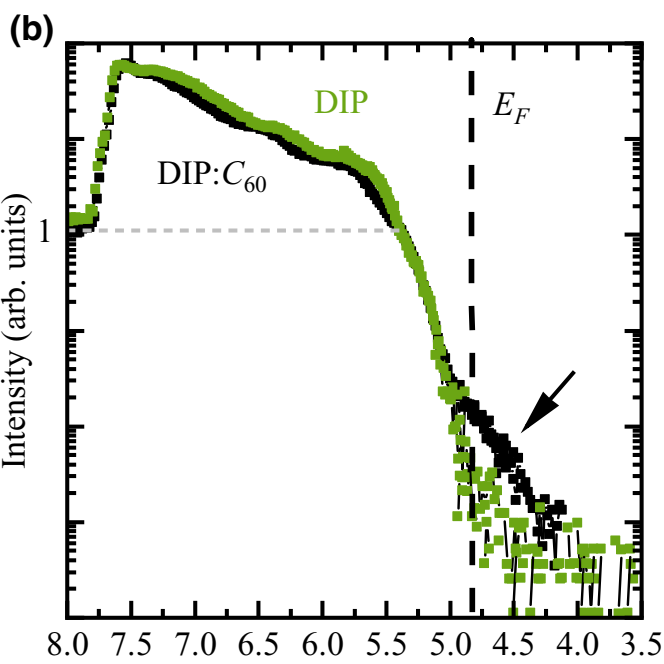

Binding energy relative to $\mathrm{VL}(\mathrm{eV})$

FIG. 2. (a) Comparison of the combined LE UPS spectra for neat DBP (blue) and a DBP: $\mathrm{C}_{60} 1: 1$ mixture (black). (b) The same for a neat DIP layer (green) and a DIP: $\mathrm{C}_{60}$ 1:1 mixture (black). In both cases, the spectra of single donor layers and D:A mixtures are normalized in intensity such that the fitted HOMO peaks (see the Supplemental Material for details) are identical, as indicated by the gray dashed line. The pronounced exponential tail structure in the DIP: $\mathrm{C}_{60}$ mixture is visualized by an arrow. $E_{F}$ denotes the Fermi energy of the respective sample. energies of about 5.8-5.9 eV relative to the vacuum level and the $\sigma$ values, i.e., the standard deviations of the Gaussian functions, are about $0.25 \mathrm{eV}$ for both neat donor layers and slightly lower at about $0.23 \mathrm{eV}$ for the mixtures with $\mathrm{C}_{60}$. This probably reflects the circumstance that dielectric screening is changed upon mixing the perylene derivatives with the higher dielectric constant fullerene. In general, $\sigma_{\text {UPS }}$ contains several contributions as discussed in Ref. [31] including, inter alia, static, and dynamic disorder. However, the obtained numbers cannot directly be compared with bulk values, e.g., derived from modeling charge transport, because a weaker screening at the surface of the films systematically broadens photoemission peaks [32].

Furthermore, in the case of the DIP: $\mathrm{C}_{60}$ mixture there is a clear deviation from a Gaussian function above the Fermi level, i.e., in the gap of the D:A mixture, as indicated by the arrow in Fig. 2(b). The almost linear decay in this semilogarithmic plot suggests the presence of exponential tail states. A closer look at the raw data in the Supplemental Material shows that such tail states are also present to some extent in neat DIP and in the DBP: $\mathrm{C}_{60}$ blend as well, but they are strongest, if DIP is coevaporated with $\mathrm{C}_{60}$. Similar exponential gap states have been observed in the literature for $\mathrm{CuPc}$, rubrene, and thick layers of neat $\mathrm{C}_{60}$ $[27,30]$. The increased density of tail states for the mixture of DIP and $\mathrm{C}_{60}$ compared to the neat DIP layer could as well originate from the $\mathrm{C}_{60}$ LUMO states that are occupied due to a charge transfer from excited DIP molecules. However, if this were the case similar results for DBP mixtures should be measured, which is not observed. Thus, a plausible explanation, which takes the morphological differences between the two donor materials into account, is that these tail states are the result of grain boundaries. In the crystalline DIP, which undergoes strong phase separation, when codeposited with $\mathrm{C}_{60}$, one can envision the coexistence of different local environments. As already briefly mentioned in the morphology section, DIP forms relatively large crystalline domains in mixtures with $\mathrm{C}_{60}$, which extend in vertical direction throughout the whole film. However, in lateral direction DIP molecules at grain boundaries will expose their $\pi$-electron system to $\mathrm{C}_{60}$ molecules having different polarizability, thus, transferring spectral weight from the main DIP HOMO peak to these exponential tail states.

Note that the origin of subgap states in polymers has recently been discussed in the context of waterinduced trap states [33,34]. While the detailed mechanism might differ in solution-cast polymer films and vacuum-evaporated molecular systems studied here, the idea of water penetrating into nanovoids and changing the dielectric screening might at least qualitatively explain the differences between the dense amorphous DBP: $\mathrm{C}_{60}$ mixtures on one side and the rough polycrystalline DIP: $\mathrm{C}_{60}$ films on the other side with its grain boundaries allowing for the permeation of such species. 


\section{Photocurrent and electroluminescence spectra}

In order to analyze whether these exponential tail states observed in the UPS spectra of DIP samples influence the device performance of organic solar cells, it is interesting to investigate their contribution to the photocurrent of the respective materials. Therefore, sensitive incident photon-to-current efficiency measurements are used to analyze particularly the influence of subgap states on the photocurrent of organic solar cells.

IPCE spectra of DIP and DBP as well as $\mathrm{C}_{60}$ single-layer devices [with the following structure: ITO/HIL 1.3/neat donor or fullerene layer $(50 \mathrm{~nm}) / \mathrm{BCP}(5 \mathrm{~nm}) / \mathrm{Al}]$ are shown in a logarithmic representation in Fig. 3 (dashed lines). The general shape of the curves is determined by the absorption spectra (shown in Fig. SI1 of the Supplemental Material [23]). However, owing to differences in the location where exciton dissociation and, thus, carrier generation happens and the disparities in carrier mobility of the materials, there are noticeable differences in the absolute magnitude of the photocurrent. The $\mathrm{C}_{60}$ device yields the highest IPCE among these single-layer devices, because the active junction is located at the interface to the HIL1.3 layer through which the device is illuminated. Additionally, $\mathrm{C}_{60}$ has the highest carrier mobility of the three materials. Comparing DIP and DBP, the higher photocurrent for the former is striking in view of the much lower absorption coefficient. However, because of the amorphous nature of DBP films their exciton diffusion

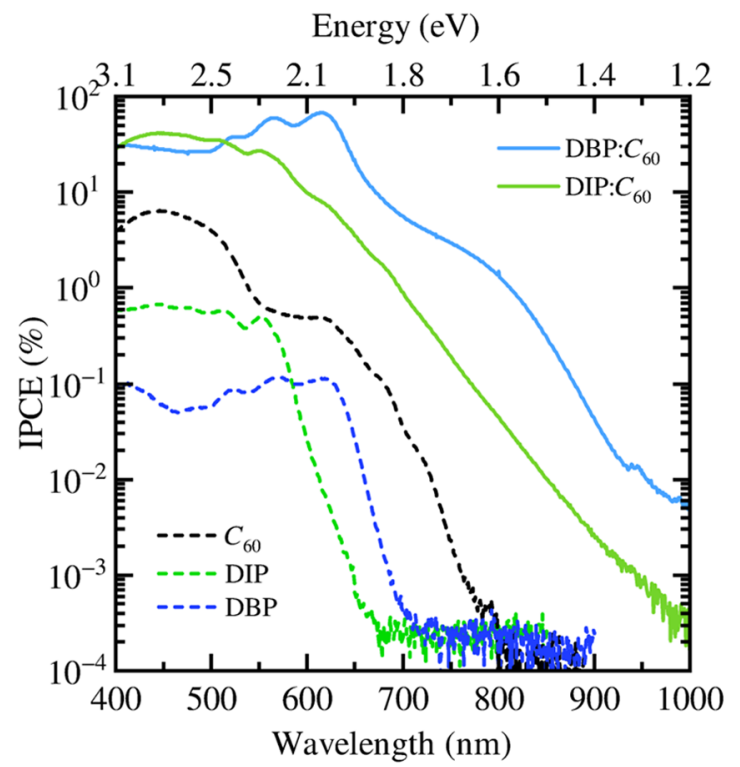

FIG. 3. IPCE spectra of $\mathrm{C}_{60}$, DIP, and DBP single-layer devices with the architecture ITO/HIL 1.3/organic layer $(50 \mathrm{~nm}) / \mathrm{BCP}(5$ $\mathrm{nm}) / \mathrm{Al}$ in comparison to DIP:C $\mathrm{C}_{60}(2: 1)$ and $\mathrm{DBP}: \mathrm{C}_{60}(1: 2)$ planar-mixed heterojunction devices with the layer sequence: ITO/PEDOT:PSS/donor(5 nm)/D:A mixture $(50 \mathrm{~nm}) / \mathrm{C}_{60}(10$ $\mathrm{nm}) / \mathrm{BCP}(5 \mathrm{~nm}) / \mathrm{Al}$. length and charge carrier mobility are significantly lower so that only a small fraction of the generated excitons is actually dissociated and extracted as charges. Furthermore, since the active junction for both donors is located at the cathode side to the BCP layer, at the given film thickness of $50 \mathrm{~nm}$ an internal filter effect may also play a role in the case of DBP (as evidenced, e.g., by the fact that the relative contribution of the 0-0 transition in the photocurrent is lower than in absorption). The crystalline DIP, on the other hand, has large exciton diffusion length (only limited by the film thickness [35]) so that almost all excitons can reach the dissociating interface and contribute to the photocurrent.

Also shown in Fig. 3 (solid lines) are IPCE spectra of DA heterostructures in a so-called planar-mixed heterojunction (PM HJ) configuration, i.e., the D:A blend (50 $\mathrm{nm}$ ) is enclosed by $5-\mathrm{nm}$ neat donor and $10-\mathrm{nm}$ neat $\mathrm{C}_{60}$. Both devices have similar IPCE in the dominant spectral range of the fullerene absorption (400-500 nm), which is about five times higher than for the single-layer $\mathrm{C}_{60}$ device. Above $500 \mathrm{~nm}$, however, huge differences between the two donor materials as well as to the single-layer devices become apparent. The DBP: $\mathrm{C}_{60}$ device shows a strong donor contribution in the range of the DBP absorption $(500-650 \mathrm{~nm})$ with the typical vibronic structure, while for the DIP: $\mathrm{C}_{60}$ device the donor only adds little to the $\mathrm{C}_{60}$ IPCE with a noticeable contribution between 500 and $550 \mathrm{~nm}$. In the longer wavelength region, the IPCE of the DIP: $\mathrm{C}_{60} \mathrm{PM} \mathrm{HJ}$ remains higher than for neat $\mathrm{C}_{60}$ in the so-called subgap region [in this case the fullerene has the lower gap of about $1.9 \mathrm{eV}(650 \mathrm{~nm})]$ and exhibits an almost exponential decay down to values of some $10^{-4}$. For the DBP: $\mathrm{C}_{60}$ device we also observe an additional contribution in the subgap region (DBP and $\mathrm{C}_{60}$ have basically the same gap). But owing to the different film morphology as compared to DIP: $\mathrm{C}_{60}$, the DBP: $\mathrm{C}_{60}$ PM HJ has significantly higher IPCE than its DIP counterpart. As will be discussed further below, this can be related to different electronic couplings of the related CT states (standing vs lying donor molecules) as well as the blend morphology (phase separation vs miscibility).

For electroluminescence spectroscopy the organic solar cell is operated as a light-emitting diode by applying a forward voltage to the device and detecting the emission. Figure 4 shows EL spectra for the single-layer devices (left) of all used materials as well as for the DBP: $\mathrm{C}_{60}$ (center) and DIP:C $\mathrm{C}_{60}$ (right) PM HJs. To understand the emission spectrum of a DA heterostructure, the spectra of the individual materials are essential. Furthermore, in order to investigate possible effects of state filling we measure each single-layer EL spectrum at different drive voltages: once at the lowest possible value that still yields a reasonable signal-to-noise ratio and then at higher voltages of 2 and $4 \mathrm{~V}$. Generally, the lowest HOMO-LUMO transition in $\mathrm{C}_{60}$ molecules is symmetry forbidden [36], however, 


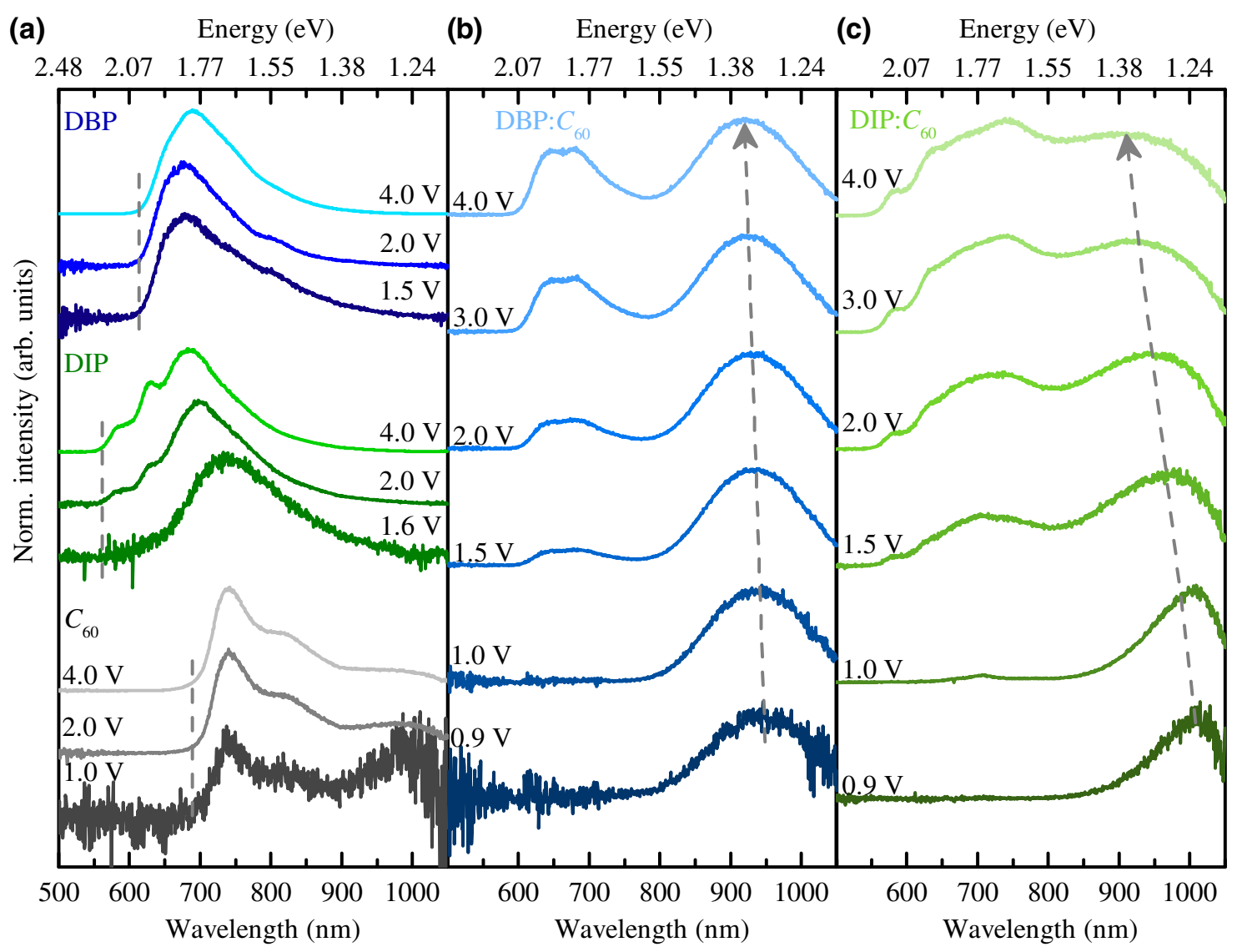

FIG. 4. Electroluminescence spectra of DBP, DIP, and $\mathrm{C}_{60}$ single-layer devices (a), as well as DBP:C $\mathrm{C}_{60}$ (b) and DIP:C $\mathrm{C}_{60}$ (c) PM HJ solar cells for different applied voltages between 0.9 and $4.0 \mathrm{~V}$. The measurements are performed at room temperature. Please note, that the rolloff above $1020 \mathrm{~nm}$ for the DIP: $\mathrm{C}_{60}$ spectra may originate from the limited sensitivity of the used Si detector. Thus, this range is not further considered in the following analysis.

vibronic coupling and disorder in thin films allow violation of the parity selection rule [37]. The main emission of $\mathrm{C}_{60}$ (gray and black lines) is located between 700 and about $900 \mathrm{~nm}[38]$ and does not change for different applied voltages; but for $1 \mathrm{~V}$ an additional weak emission appears in the longer wavelength region. This contribution is generally not discussed in the literature so that a clear assignment is not possible. Similar observations are made for the DBP single-layer device (blue lines), where the spectra for $1.5,2$, and $4.0 \mathrm{~V}$ almost perfectly match each other, although not showing clearly resolved vibronic structure in this case. The spectral shape for the DIP emission (green lines), however, strongly depends on the applied voltage. For low voltages, a broad featureless spectrum with a maximum at about $740 \mathrm{~nm}$ is measured. Increasing the applied voltage to $2 \mathrm{~V}$ leads to a blue shift by about 50 $\mathrm{nm}$ and a pronounced vibronic structure, especially for shorter wavelengths, appears, which becomes more pronounced upon increasing the voltage to $4 \mathrm{~V}$. However, a weak contribution of the broad featureless emission at the same position as the spectrum for low applied voltage is still present. The main EL peak positions are similar to those found by photoluminescence measurements in the literature $[8,39,40]$.

For the PM HJ devices of both donors, the EL spectra show a distinct dependence on the applied voltage. Firstly, for low voltages just above $V_{\mathrm{OC}}$ only a broad signal in the long-wavelength range is detected. As the single materials show no relevant signal at these wavelengths, this emission is attributed to CT states at the DA interface. However, the higher the applied voltage gets the more of the original bulk emission of the donor at shorter wavelengths contributes. Secondly, the long-wavelength bands assigned to emission from $\mathrm{CT}$ states show a distinct difference in their peak positions for both donor materials. Both of them shift toward shorter wavelength with increasing voltage, as indicated by the dashed arrows. However, the magnitude of the shift is largely different. For DBP: $\mathrm{C}_{60}$ the $\mathrm{CT}$ maximum shifts by only about $25 \mathrm{~nm}$ over the whole voltage range, whereas the shift for the DIP: $\mathrm{C}_{60}$ blend is more than 100 $\mathrm{nm}$. As discussed in the following sections, this indicates significant differences in their CT DOS. 


\section{ANALYSIS AND DISCUSSION}

According to fundamental thermodynamic principles the absorption and emission spectra of a solar cell are linked by reciprocity relations [41]. In particular, if absorption and emission are between states that are populated according to Boltzmann statistics, which is an approximation, the respective spectra are related by

$$
\operatorname{Emission}(E) \propto \operatorname{Absorption}(E) \times E^{2} \times \exp \left(-\frac{E}{k_{B} T}\right),
$$

whereby suitable spectral quantities have to be used for "Emission" and "Absorption," respectively.

In the context of organic solar cells it has become common practice to take electroluminescence spectra on the one side, and external quantum efficiency (EQE), i.e., IPCE, spectra on the other side [42]; although some authors have recently suggested to use PL instead of EL [14]. Note that the conversion of measured EL spectra using a wavelength-dispersive spectrometer [yielding intensities in $\mathrm{W} /\left(\mathrm{cm}^{2} \mathrm{~nm}\right)$ ] to reduced EL spectra not only requires converting wavelength to energy (and, concomitantly, dividing intensity by a factor $E^{2}$ ), but needs explicit division by another factor $E$. On the other hand, for obtaining reduced IPCE spectra, which is a dimensionless quantity, multiplication with a factor $E$ is sufficient.

In the following, we use the methodology put forward by Vandewal et al. on how to determine optical gaps in organic photovoltaic materials [42], which was recently challenged by the work of Kahle et al., who suggested a different approach to interpret absorption and emission spectra of CT states in such systems [14]. We start with the analysis of single-layer devices and demonstrate that both perylene donors exhibit distinct differences concerning their DOS, which is thereafter found to cause different scenarios in DA solar cells as well.

\section{A. Single-donor devices}

The shape of absorption and emission spectra of organic thin films is commonly described using a Franck-Condon progression of vibrational modes around the optical gap $E_{\text {opt }}$, which is eventually multiplied by a Gaussian function $\left[\Gamma(E)=\left(2 \pi \sigma^{2}\right)^{-1 / 2} \exp \left(-E^{2} / 2 \sigma^{2}\right)\right.$ with variance $\left.\sigma^{2}\right]$ to account for inhomogenous line broadening due to static disorder [14].

Then the reduced EL spectrum $\tilde{I}_{\mathrm{EL}}(E)$ is obtained by dividing the EL spectrum $I_{\mathrm{EL}}(E)$ by the energy $E$, and the reduced IPCE spectrum $\tilde{\eta}_{\text {IPCE }}(E)$ by multiplying the IPCE spectrum $\eta_{\mathrm{IPCE}}(E)$ with $E$ :

$$
\begin{aligned}
\tilde{I}_{\mathrm{EL}}(E) & \equiv \frac{I_{\mathrm{EL}}(E)}{E} \\
& \propto \sum_{m} \frac{S^{m}}{m !} \exp (-S) \times \Gamma\left[E-\left(E_{0}-m \hbar \omega_{\mathrm{vib}}\right)\right],
\end{aligned}
$$

$$
\begin{aligned}
\tilde{\eta}_{\mathrm{IPCE}}(E) & \equiv \eta_{\mathrm{IPCE}}(E) \times E \\
& \propto \sum_{m} \frac{S^{m}}{m !} \exp (-S) \times \Gamma\left[E-\left(E_{0}+m \hbar \omega_{\mathrm{vib}}\right)\right] .
\end{aligned}
$$

Therein $E_{0}$ is the energy of the purely electronic 0-0 transition, $\omega_{\text {vib }}$ is the frequency of the intramolecular vibration to which the electronic excitation couples predominantly, and $S$ is the Huang-Rhys factor indicating which vibrational quantum number $(m=0,1,2, \ldots)$ is most likely, i.e., how large the difference in the equilibrium positions between ground- and excited-state potential minima is.

This formalism is now applied to reduced IPCE and EL spectra of DIP and DBP single-layer devices. Figure 5 shows normalized reduced photocurrent action spectra of both donors on a logarithmic scale. The first peak of the DBP IPCE spectrum at $E=2.01 \mathrm{eV}$ can be fitted with one single Gaussian having $\sigma=56.2 \mathrm{meV}$, whereas for the DIP spectrum a shoulder at lower energies is visible, so that two Gaussians at $E_{2}=2.25 \mathrm{eV}$ and $E_{1}=2.15 \mathrm{eV}$ with $\sigma_{2}=59.0 \mathrm{meV}$ and $\sigma_{1}=41.4 \mathrm{meV}$, respectively, are necessary. In both cases, the energy of the dominant peak is assigned to the electronic 0-0 transition, while the second weaker peak in DIP probably results from a Davidov splitting, because the crystalline DIP phase has two molecules per unit cell [43].

Please note that the $\sigma$ values obtained from UPS and optical spectra are not expected to be identical. As already discussed in the section on photoelectron spectroscopy, $\sigma_{\text {UPS }}$ does not quantitatively reflect the width of the DOS because it is broadened due to specific issues of this method. Furthermore, one has to keep in mind that the width of the DOS is larger for charged organic molecules than for neutral excited species. Typically, it is found that $\sigma_{\text {charge }} \approx 2.5 \times \sigma_{\text {exciton }}$ [45]. Using this relation, a width of the transport DOS between 140 and $150 \mathrm{meV}$ is expected for DIP and DBP. It is also remarkable that the $\sigma$ values are almost identical for both donors in spite of the fact that DIP grows as polycrystalline thin films, whereas DBP films are amorphous.

Nevertheless, they exhibit significant differences in the so-called subgap region at energies below their respective optical gaps. For DBP only a small deviation of the measured spectrum from the Gaussian fit at the lowest energies is observed. But still, the fitted straight line indicates the presence of a small amount of exponential tail states. In the case of DIP, however, the deviation from the Gaussian fit is much larger. For energies below about $2.0 \mathrm{eV}$ a pronounced exponential contribution of the IPCE spectrum can be observed. From the slope of these linear fits an Urbach energy $E_{U}$ characterizing the energetic depth of the tail states can be calculated. It amounts to about $50 \mathrm{meV}$ for DIP, but is significantly smaller (about $31 \mathrm{meV}$ ) for DBP, 
(a)

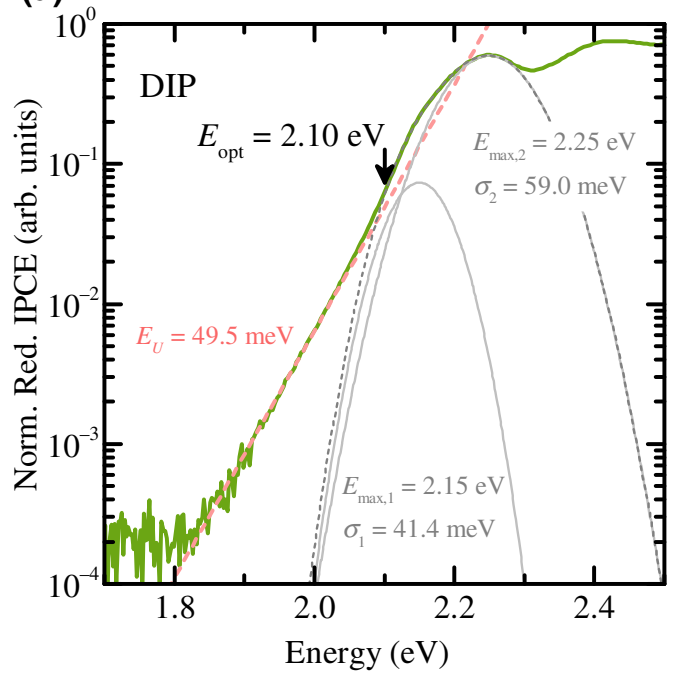

(b)

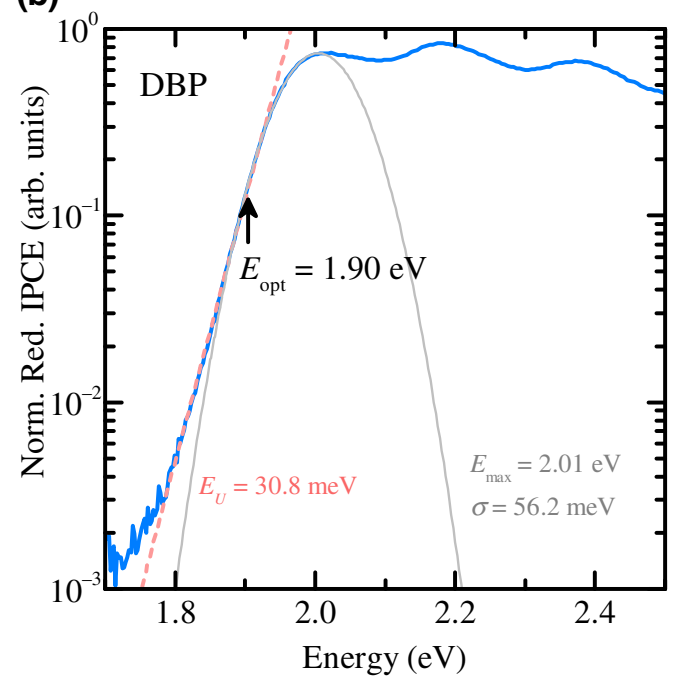

FIG. 5. Reduced normalized IPCE spectra of DIP (a) and DBP (b) single-layer devices with the architecture ITO/HIL 1.3/organic layer $(50 \mathrm{~nm}) / \mathrm{BCP}(5 \mathrm{~nm}) / \mathrm{Al}$. The first absorption peak of DBP is well described with one Gaussian only, whereas for DIP two Gaussians are necessary. The low-energy region is fitted linearly to emphasize the presence of tail states, most pronounced in DIP. From the slopes an Urbach energy $E_{U}$ is calculated. The literature values for the optical gaps $E_{\text {opt }}$ of $2.1 \mathrm{eV}$ for DIP [43] and 1.9 eV for DBP [44] are marked with black arrows.

which is comparable to high-performance polymer solar cells [46].

We now turn to the combined analysis of EL and IPCE spectra where, in particular, the voltage dependence of the EL will yield further information. Figure 6 shows normalized reduced EL and IPCE spectra of the two donor single-layer devices on linear scale. In both cases, the IPCE spectra exhibit a well-resolved vibronic structure, which can be described by a Franck-Condon formalism. From the analysis shown in Fig. SI6 and Table SI-II of the Supplemental Material [23], similar vibronic progressions $\hbar \omega_{\text {vib }}$ between about 175 and $185 \mathrm{meV}$ are obtained, and the inhomogenous linewidth is almost $70 \mathrm{meV}$ in both cases - somewhat larger than in the analysis made in Fig. 5, where the focus is on the low-energy slope only.

By contrast, the EL spectra of both donors are affected by aggregate formation such that the apparent line width does not reflect the intrinsic inhomogenous line broadening. As shown in the Supplemental Material [Fig. SI6(a) [23] ], the EL of DBP can only be measured with vibronically resolved progression, if DBP is diluted in rubrene as host material. Since both are nonpolar and have similar dielectric constants, the excitonic energies of DBP should be identical in both cases. Dilute DBP in rubrene has its 0 0 transition slightly above $2 \mathrm{eV}$, i.e., more or less directly at the $0-0$ transition in the IPCE with negligible Stokes shift, and the $0-1$ transition is found at $1.86 \mathrm{eV}$. However, in the EL of the neat DBP film shown in Fig. 6(b) the 0-0 transition is suppressed, probably due to self-absorption. Rather, a broad peak with its maximum at about $1.78 \mathrm{eV}$ is observed, which is probably composed of an aggregate emission together with the $0-1$ and $0-2$ peaks (see the Supplemental Material for a detailed analysis) [23]. This is similar in the case of DIP, where the $0-0$ transition is also completely suppressed and the EL spectrum is dominated by a broad peak at lower energies.

However, there is a striking difference between both donors. In the case of the amorphous DBP there is basically no difference on the high-energy side of the EL spectra measured at 1.5, 2, and even $4 \mathrm{~V}$ [Fig. 6(a)]. Only at the low-energy side of the spectra can one observe some weak additional contribution for the lowest voltage, probably due to the above mentioned tail states. This is in contrast to the DIP EL spectra, where the low-voltage spectrum measured at $1.6 \mathrm{~V}$ is shifted as a whole by almost $200 \mathrm{meV}$ towards lower energy [Fig. 6(b)]. Furthermore, this spectrum is almost featureless and does not have a vibronic substructure. By contrast, as soon as the voltage is increased to $2 \mathrm{~V}$ the expected vibronic features of the $0-1$ and $0-2$ transitions at about 2.1 and $1.95 \mathrm{eV}$, respectively, appear and do not change significantly for higher voltages. Thus, one has to conclude that at the lowest applied voltage of $1.6 \mathrm{~V}$ (which is about $0.5 \mathrm{~V}$ below the optical gap) the EL of polycrystalline neat DIP devices is completely dominated by defect luminescence from lower lying states in the DOS.

\section{B. Donor-acceptor heterojunction devices}

Following Marcus theory for electron transfer reactions, Vandewal et al. have put forward the following 
(a)

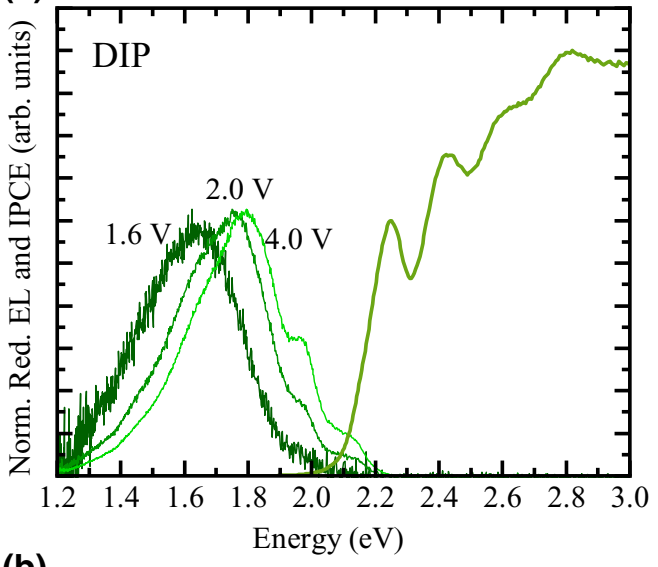

(b)

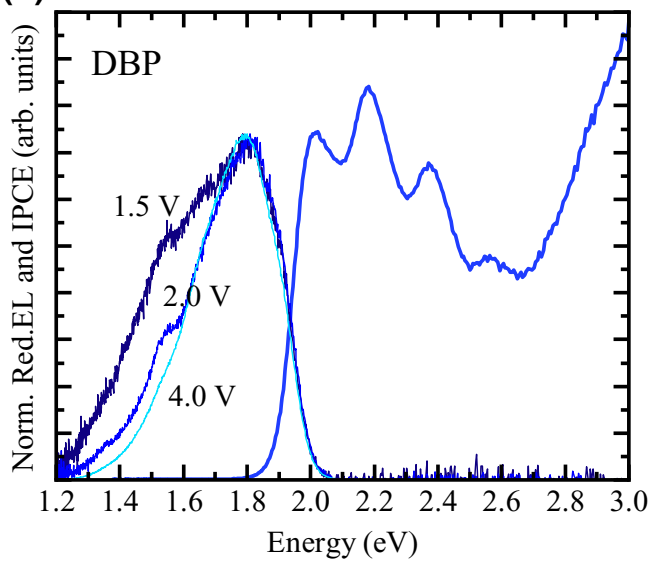

FIG. 6. Reduced normalized EL and IPCE spectra of DIP (a) and DBP (b) single-layer devices with the architecture ITO/HIL 1.3/organic layer $(50 \mathrm{~nm}) / \mathrm{BCP}(5 \mathrm{~nm}) / \mathrm{Al}$. A detailed analysis of the spectra using the Franck-Condon formalism is shown in the Supplemental Material.

expressions for the high-energy side of the reduced EL as well as the low-energy part of the reduced IPCE spectra originating from CT states in DA solar cells $[4,5]$ :

$$
\begin{aligned}
\tilde{I}_{\mathrm{EL}}(E) \propto & \frac{1}{\sqrt{4 \pi \lambda_{\mathrm{DA}} k_{B} T}} \\
& \times \exp \left(-\frac{\left[E-\left(E_{\mathrm{CT}}-\lambda_{\mathrm{DA}}\right)\right]^{2}}{4 \lambda_{\mathrm{DA}} k_{B} T}\right) \\
\tilde{\eta}_{\mathrm{IPCE}}(E) \propto & \frac{1}{\sqrt{4 \pi \lambda_{\mathrm{DA}} k_{B} T}} \\
& \times \exp \left(-\frac{\left[E-\left(E_{\mathrm{CT}}+\lambda_{\mathrm{DA}}\right)\right]^{2}}{4 \lambda_{\mathrm{DA}} k_{B} T}\right) .
\end{aligned}
$$

These are Gaussian functions, where the CT energy $E_{\mathrm{CT}}$ is the crossing point of properly normalized reduced EL and IPCE spectra, and the reorganization energy $\lambda_{\text {DA }}$ determines the Stokes shift between their maxima (being equal to $2 \lambda_{\mathrm{DA}}$ ) and the width of both spectra (with the variance $w^{2}$ given by $\left.2 \lambda_{\mathrm{DA}} k_{B} T\right)$ [4]. It is important to note that $\lambda_{\mathrm{DA}}$, i.e., the reorganization energy between the ground and excited CT state, contains contributions of both the donor and acceptor molecules [47]. It is thought to originate from low-frequency intermolecular modes of the DA pair forming the CT state ("dynamic disorder") that have energies significantly less than the thermal energy, which results in a Boltzmann-type occupation of these modes [42]. Furthermore, in contrast to the Franck-Condon description from above, this Marcus-type expression does not contain any contribution of static disorder arising, e.g., from the coexistence of many different electronic configurations at the DA interface.

To account for this, Burke et al. have suggested to modify these expressions by introducing a CT disorder parameter $\sigma_{\mathrm{CT}}$ [9], such that the experimentally obtained values for $E_{\mathrm{CT}}^{\exp }$ and $\lambda_{\mathrm{CT}}^{\exp }$ are linked to their "intrinsic" values by

$$
\begin{aligned}
& E_{\mathrm{CT}}^{\exp }=E_{\mathrm{CT}}-\frac{\sigma_{\mathrm{CT}}^{2}}{2 k_{B} T} \quad \text { and } \\
& \lambda_{\mathrm{CT}}^{\exp }=\lambda_{\mathrm{CT}}+\frac{\sigma_{\mathrm{CT}}^{2}}{2 k_{B} T} .
\end{aligned}
$$

Following the reasoning in Ref. [9], this results in slightly modified expressions for the reduced EL and IPCE lineshapes:

$$
\begin{aligned}
\tilde{I}_{\mathrm{EL}}(E) \propto & \frac{1}{\sqrt{2 \pi\left(\sigma_{\mathrm{CT}}^{2}+2 \lambda_{\mathrm{CT}} k_{B} T\right)}} \\
& \times \exp \left\{-\frac{\left[E-\left(E_{\mathrm{CT}}-\lambda_{\mathrm{CT}}-\frac{\sigma_{\mathrm{CT}}^{2}}{k_{B} T}\right]^{2}\right.}{4 \lambda_{\mathrm{CT}} k_{B} T+2 \sigma_{\mathrm{CT}}^{2}}\right\}, \\
\tilde{\eta}_{\mathrm{IPCE}}(E) \propto & \frac{1}{\sqrt{2 \pi\left(\sigma_{\mathrm{CT}}^{2}+2 \lambda_{\mathrm{CT}} k_{B} T\right)}} \\
& \times \exp \left\{-\frac{\left[E-\left(E_{\mathrm{CT}}+\lambda_{\mathrm{CT}}\right)\right]^{2}}{4 \lambda_{\mathrm{CT}} k_{B} T+2 \sigma_{\mathrm{CT}}^{2}}\right\} .
\end{aligned}
$$

Please note that the expression (7) was originally not derived by Burke et al. However, if the redefinition of $E_{\mathrm{CT}}$ and $\lambda_{\mathrm{CT}}$ from Eq. (6) and reciprocity are used, it follows that the expression for the EL spectrum should take this form. As a consequence, the Stokes shift between both spectra does now also contain a contribution of disorder and amounts to $2 \lambda_{\mathrm{CT}}+\sigma_{\mathrm{CT}}^{2} / k_{B} T$, just like the variance, which is now given by $w^{2}=2 \lambda_{\mathrm{CT}} k_{B} T+\sigma_{\mathrm{CT}}^{2}$.

A different approach to include static disorder has been put forward by Kahle et al. using a Franck-Condon formalism [14] to describe reduced PL and IPCE spectra. However, for the low-frequency intermolecular modes relevant 
(a)

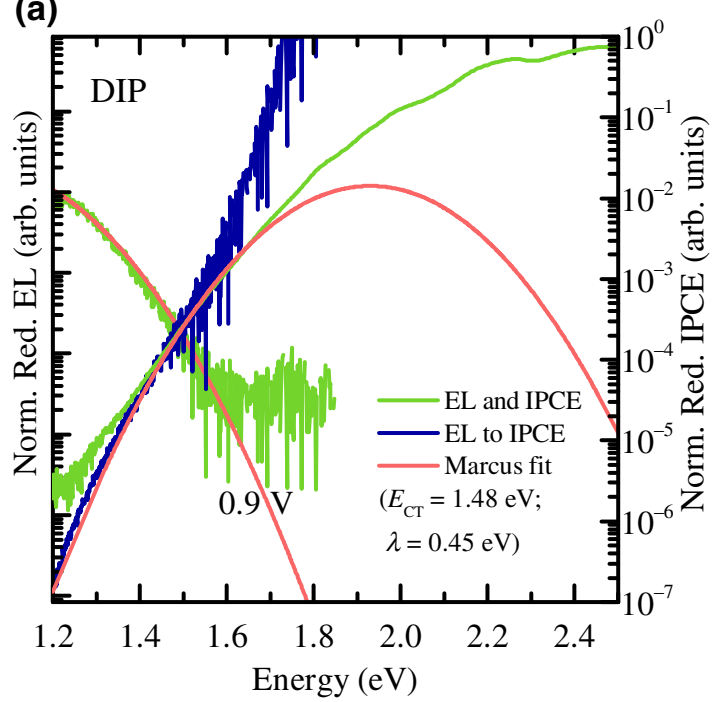

(b)

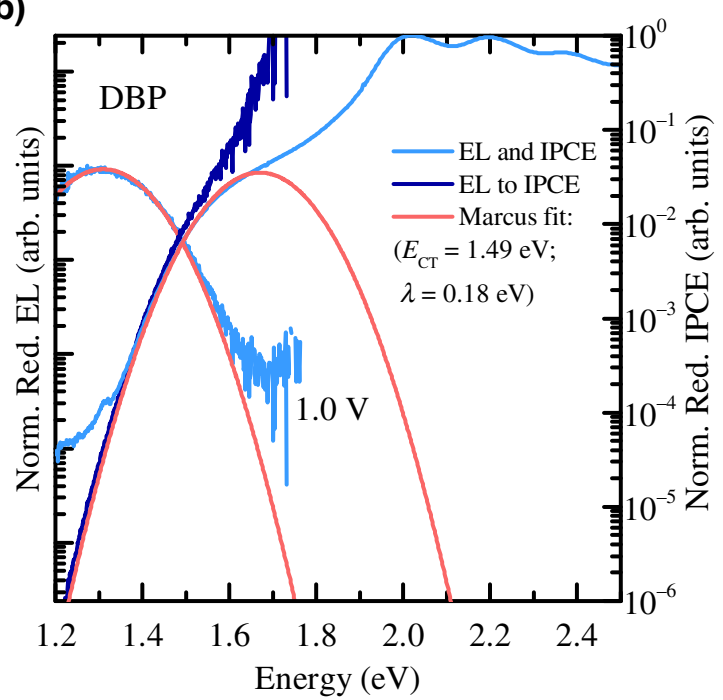

FIG. 7. Reduced CT absorption (IPCE) and emission (EL) spectra of DIP:C $\mathrm{C}_{60}$ (a) and DBP: $\mathrm{C}_{60}$ (b) and fits with Gaussian line shapes according to Marcus theory as proposed by Vandewal et al. [4,5]. The fit parameters are given in Table I. In addition, the EL spectra are converted into absorption using the reciprocity relation (1).

for CT states, this also leads to Gaussian line shapes:

$$
\begin{aligned}
\tilde{I}_{\mathrm{PL}}(E) \propto & \frac{1}{\sqrt{4 \pi \lambda_{\text {low }} k_{B} T}} \\
& \times \exp \left\{-\frac{\left[E-\left(E_{\mathrm{CT}}-\lambda_{\text {low }}\right)\right]^{2}}{4 \lambda_{\text {low }} k_{B} T+2 \sigma^{2}}\right\}, \\
\tilde{\eta}_{\mathrm{IPCE}}(E) \propto & \frac{1}{\sqrt{4 \pi \lambda_{\text {low }} k_{B} T}} \\
& \times \exp \left\{-\frac{\left[E-\left(E_{\mathrm{CT}}+\lambda_{\text {low }}\right)\right]^{2}}{4 \lambda_{\text {low }} k_{B} T+2 \sigma^{2}}\right\} .
\end{aligned}
$$

Thus, the Stokes shift is now only $2 \lambda_{\text {low }}$, but the variance again contains a contribution of static disorder: $w^{2}=$ $2 \lambda_{\text {low }} k_{B} T+\sigma^{2}$. It is argued that the smaller Stokes shift as compared to Burke et al. reflects the fact that EL spectra are shifted to lower energy as compared to PL, because the former include a contribution from spectral relaxation [14].

We analyze our data mainly using the expressions (4) and (5) and (7) and (8), however, by considering the alternative description (9) and (10) as well.

For DA heterojunction solar cells the analysis requires using a semilogarithmic representation of reduced absorption and emission spectra, because the contribution to the photocurrent originating from CT states is located in the subgap region and is significantly lower than the main absorption features of the neat materials. Figure 7 shows normalized spectra together with fits according to Marcus theory. For the devices with DBP as donor, the CT contribution to the IPCE spectrum in the subgap region (i.e., below $1.9 \mathrm{eV}$ ) is clearly visible and has its pendant in the EL emission at lower energies, which is normalized to the same peak height. Both can well be fitted by Gaussian line shapes according to Eqs. (4) and (5) yielding fit parameters of $E_{\mathrm{CT}}=1.49 \mathrm{eV}$ and $\lambda_{\mathrm{DA}}=0.18 \mathrm{eV}$ [Fig. 7(b)], which are also listed in Table I for comparison with other models. Furthermore, we use the reciprocity between absorption and emission according to Eq. (1) to convert the measured EL spectrum to a photocurrent signal. As shown in Fig. 7(b), the EL spectrum corresponds (after proper multiplication with a scaling factor) very well to the IPCE spectrum for $E \leq E_{\mathrm{CT}}$. In addition to the EL spectrum obtained at an applied voltage of $1.0 \mathrm{~V}$, we also obtain spectra up to $4 \mathrm{~V}$ (shown in the Supplemental Material, Fig. SI7 [23]). However, there is very little shift between them yielding basically the same CT and reorganization energies.

This is in stark contrast to the DIP: $\mathrm{C}_{60}$ devices, where the contribution of CT states to the IPCE spectra is more smeared out and, accordingly, not very well described by

TABLE I. Compilation of CT energy $E_{\mathrm{CT}}$, reorganization energy $\lambda$ and disorder parameter $\sigma$ from fitting CT spectra of

\begin{tabular}{|c|c|c|c|c|c|}
\hline \multirow[b]{2}{*}{ Device } & \multicolumn{2}{|c|}{ Marcus } & \multicolumn{3}{|c|}{ Burke } \\
\hline & $\begin{array}{l}E_{\mathrm{CT}} \\
(\mathrm{eV})\end{array}$ & $\begin{array}{l}\lambda \mathrm{DA} \\
(\mathrm{eV})\end{array}$ & $\begin{array}{l}E_{\mathrm{CT}} \\
(\mathrm{eV})\end{array}$ & $\begin{array}{l}\lambda_{\mathrm{CT}} \\
(\mathrm{eV})\end{array}$ & $\begin{array}{c}\sigma_{\mathrm{CT}} \\
(\mathrm{meV})\end{array}$ \\
\hline \multirow{2}{*}{$\mathrm{DIP}: \mathrm{C}_{60}$} & 1.48 & 0.45 & 1.55 & 0.38 & 59 \\
\hline & & & 1.75 & 0.18 & 116 \\
\hline $\mathrm{DBP}: \mathrm{C}_{60}$ & 1.49 & 0.18 & 1.55 & 0.12 & 56 \\
\hline
\end{tabular}
DIP: $\mathrm{C}_{60}$ and DBP: $\mathrm{C}_{60}$ solar cells according to the Marcus formalism [Eqs. (4) and (5)] as well as the Burke model [Eqs. (7) and (8)]. 
a Gaussian [Fig. 7(a)]. It rather appears that there is an additional exponential contribution, most likely originating from tail states as already discussed in the context of photoemission spectroscopy and single-donor devices. Moreover, if the EL spectra at an applied voltage of $0.9 \mathrm{~V}$ (i.e., at $V_{\mathrm{OC}}$ ) and higher voltages (see Supplemental Material and the discussion below) are compared one observes a large shift that would result in different $E_{\mathrm{CT}}$ values depending on the applied voltage. For this reason we again use the reciprocity relation [Eq. (1)] to convert the reduced EL to IPCE. As shown in Fig. 7(a), the converted EL data obtained at $V_{\mathrm{OC}}$ correspond reasonably well to the measured IPCE spectrum for $E \leq E_{\mathrm{CT}}$, whereas this would not be the case for the EL spectra at higher voltages. We therefore argue that EL spectra measured close to $V_{\mathrm{OC}}$ should be used for the extraction of the CT energy. Furthermore, the obtained value of $E_{\mathrm{CT}}=1.48 \mathrm{~V}$ agrees reasonably well with the extrapolated $V_{\mathrm{OC}}$ at $T=0 \mathrm{~K}$, (shown in Fig. SI3 of the Supplemental Material [23]), which is an established alternative method for the determination of $E_{\mathrm{CT}}$ [8].

However, while both donor materials apparently yield the same $E_{\mathrm{CT}}$, there is a striking difference in their reorganization energies (see Table I). Owing to the larger Stokes shift observed between reduced EL and IPCE spectra, the $\lambda_{\mathrm{DA}}$ value for the DIP: $\mathrm{C}_{60}$ cell $(0.45 \mathrm{eV})$ is more than twice as large as for the DBP: $\mathrm{C}_{60}$ case $(0.18$ $\mathrm{eV})$. Given the similar chemical structure of both donor molecules, one would in the first place not expect such a huge difference in their donor-acceptor reorganization energies. Indeed, density-functional-theory (DFT) calculations using the Schrödinger Materials Science software yield relatively similar reorganization energies for holes in DIP and DBP $\left(\lambda_{D}=106 \mathrm{meV}\right.$ vs $84 \mathrm{meV}$, respectively). If the reorganization energy for electrons in $\mathrm{C}_{60}$ $\left(\lambda_{A}=109 \mathrm{meV}\right.$ [48]) is added, one arrives at a total $\lambda_{\mathrm{DA}}$ of $215 \mathrm{meV}$ for DIP:C 60 and $193 \mathrm{meV}$ for DBP:C 60 , respectively. The latter is very close to the result obtained by Marcus fits to the measured CT spectra. However, because reorganization energies can also contain a contribution by the environment, which is expected to be different in amorphous vs phase-separated nanocrystalline systems, this can be seen as a direct indication for the importance of morphology for the CT DOS. Moreover, one can ask the question if, in addition to dynamic disorder, there is also a significant contribution of static disorder in these systems.

Therefore, we use the procedures put forward by Burke et al. and by Kahle et al. to analyze luminescence and photocurrent spectra of both systems and compared them to the results from the Marcus-type expressions. All of them describe Gaussian line shapes, however, they differ in the origin of the line width and the magnitude of the Stokes shift. Let us start with the Burke expression. According to Eq. (6) there is a straightforward correspondence to the "experimental" parameters $\left(E_{\mathrm{CT}}^{\exp }\right.$ and $\left.\lambda_{\mathrm{CT}}^{\exp }\right)$, which are the ones obtained from a Marcus fit. However, the introduction of a third parameter $\left(\sigma_{\mathrm{CT}}\right)$ leaves room for variations. One could, e.g., hypothetically assume that the line broadening by static disorder derived from the analysis of single-donor devices are the same in the CT spectra, although this may not necessarily be the case, because an exciton on the neat donor molecules sees a different environment than a $\mathrm{CT}$ exciton at the DA interface. Then, if $\sigma_{\mathrm{CT}}$ is fixed, the "intrinsic" values $E_{\mathrm{CT}}$ and $\lambda_{\mathrm{CT}}$ are given by Eq. (6). These parameters are listed in Table I for DIP: $\mathrm{C}_{60}$ (first line) and DBP: $\mathrm{C}_{60}$ (third line). The corresponding simulated EL and IPCE line shapes are shown in Figs. 8(a) and 8(b). Alternatively, one could postulate that DIP: $\mathrm{C}_{60}$ should have the same $\lambda_{\mathrm{CT}}$ as DBP:C $\mathrm{C}_{60}$ (in the absence of static disorder) and derive the corresponding $\sigma_{\mathrm{CT}}$ required to yield the same line shape as the Marcus fit (see Table I, second line). At room temperature all these curves are - per definition - indistinguishable. However, if temperature is lowered they will exhibit different progression of their peak positions and line widths, as will be discussed in more detail below.

Turning to the expression suggested by Kahle et al. and using the same values as in the Burke model, it is apparent from Figs. 8(a) and 8(b) that there is a large discrepancy: the simulated PL spectra are significantly higher in energy than the EL spectra from the other two approaches. The reason is that the Stokes shift in this model is given by $2 \lambda_{\text {low }} \equiv 2 \lambda_{\text {CT }}$ and does not depend on the static disorder parameter $\sigma$. The authors argue that the difference arises because PL (and not EL) spectra are used, because the latter - as already mentioned above - undergo spectral relaxation. However, in the case of DBP as donor, we doubt that this can explain the discrepancy. Considering that the DBP single-donor device [Fig. 6(b)] exhibits EL spectra with very little Stokes shift to the IPCE and that the DBP: $\mathrm{C}_{60}$ heterojunction device shows almost negligible shift of the EL spectra with voltage, we believe that spectral relaxation is not relevant in DBP-based solar cells. Furthermore, we compare EL and PL spectra originating from CT states (see Supplemental Material Fig. SI8 [23]). Although, the measured PL spectrum of DBP: $\mathrm{C}_{60}$ is shifted to higher energies (PL peak at about $1.35 \mathrm{eV}$ as compared to $1.31 \mathrm{eV}$ for EL at the lowest voltage), the shift is much less than in the simulated PL spectra according to the model by Kahle et al. Moreover, the PL spectrum shows a second peak at higher energies, which is not observed in EL. These differences in peak position and shape between EL and PL thus raise additional questions if they originate from the same states. We note that in the case of DIP: $\mathrm{C}_{60}$ the PL spectra are also shifted to higher energy and broadened with respect to the EL spectra. But even there, the shift is not nearly as large as predicted using the Kahle expression.

However, this leads us to the question of how the CT spectra of DIP: $\mathrm{C}_{60}$ with its apparent large Stokes shift and line width can be understood. We therefore turn back to 

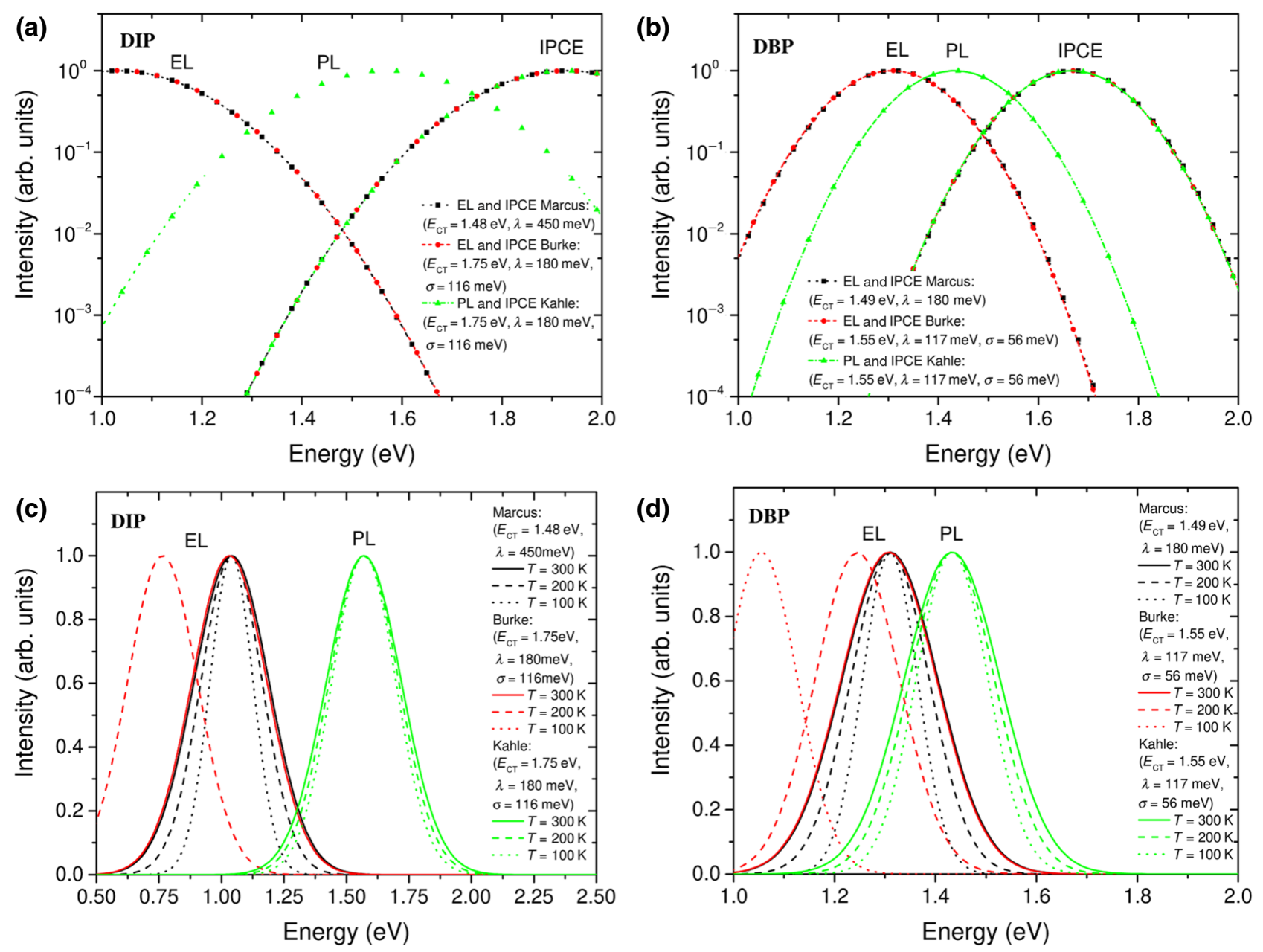

FIG. 8. Simulated EL and PL, and IPCE, spectra of CT states in DIP: $\mathrm{C}_{60}$ and DBP:C 60 solar cells. (a),(b) Results for room temperature using the expressions for the Marcus-type analysis [Eqs. (4) and (5)], the model by Burke et al. [Eqs. (7) and (8)] as well as the one by Kahle et al. [Eqs. (9) and (10)] with parameters indicated in the graphs. EL and PL spectra as a function of temperature predicted by these models are shown in (c),(d).

the voltage dependence of their EL spectra (see Fig. 4). As shown in Fig. SI9 and Table SI-III of the Supplemental Material [23], the corresponding reduced emission spectra can be fitted by Gaussians to determine their peak shift with applied voltage and, thus, current flowing through the device. The result is shown in Fig. 9. We find that the EL peak shifts by about $160 \mathrm{meV}$ over a range of 3 orders of magnitude in current density. A linear fit of the peak position to $\log j$ results in a slope of about $60 \mathrm{meV} / \mathrm{dec}$, which is almost exactly the value expected for state filling in an exponential DOS $\left[\left(k_{B} T\right) \times \ln (10)=59 \mathrm{meV} / \mathrm{dec}\right.$ at room temperature, if an ideality factor of 1 is assumed] [49]. DBP: $\mathrm{C}_{60}$, on the other hand, exhibits a very small slope of only $9.4 \mathrm{meV} / \mathrm{dec}$ in the same range, which again confirms that state filling does not play an important role in this system.

Closely related to the issue of a voltage-dependent shift of the EL spectra is the question whether the recombination of electron-hole pairs comes from fully relaxed CT states or whether it arises from a nonequilibrium occupation of the DOS. While Roland et al. have found evidence for the former scenario after photogeneration of carriers in polymer:fullerene blends [50], Melianas et al. have shown that the recombination of injected charge carriers in such systems is typically originating from states that are not fully relaxed in the DOS [16]. In our studies, we find clear evidence for a strong shift of CT EL spectra in the case of polycrystalline DIP: $\mathrm{C}_{60}$-following $\log j \propto\left(k_{B} T\right) \times \ln (10) /$ decade, which is a strong indication for state filling in an exponential DOS tail. For the amorphous DBP: $\mathrm{C}_{60}$ blends, however, we do not see such a shift. Primarily, this means that state filling does not play a significant role in that system, which could simply be a consequence of a Gaussian DOS without additional tail states. Whether or not the occupation of this DOS is in thermal equilibrium is another issue, which cannot be 


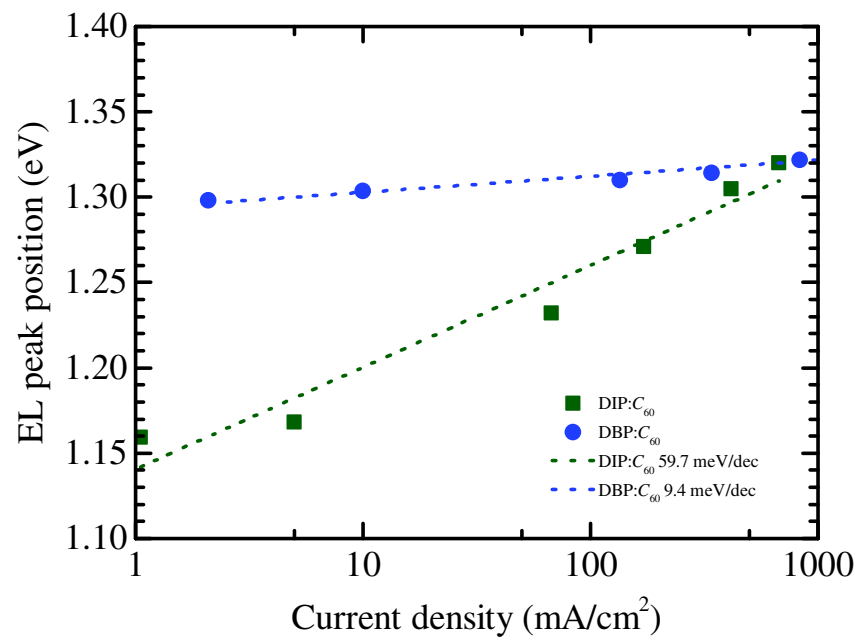

FIG. 9. Shift of the EL peak with current density for DIP: $\mathrm{C}_{60}$ and DBP: $\mathrm{C}_{60}$ solar cells. The peak positions are obtained from Gaussian fits to reduced EL spectra as detailed in the Supplemental Material. The dashed lines show linear fits to the obtained data with slopes given in $\mathrm{meV}$ per decade as indicated in the graph.

judged on the basis of our data. Nevertheless, the fact that in the case of DIP: $\mathrm{C}_{60}$ - having a DOS with tail states - we do see emission from lower lying CT states and the concomitant spectral shift upon increasing the carrier density, can be taken as indication that relaxation to lower lying states in the DOS occurs - at least to some extent. Of course, this does not necessarily mean that EL stems from fully relaxed electron-hole pairs.

Finally, we return to the question of how large the relative contributions of dynamic $(\lambda)$ and static disorder $(\sigma)$ to the CT spectra are. As shown by the above discussed simulations (Fig. 8), it is not possible to give a conclusive answer based on room-temperature spectra alone. We therefore investigate how the EL peak position and width for $\mathrm{DBP}: \mathrm{C}_{60}$, where tail states are less important, evolve as a function of temperature. According to the expression (6) suggested by Burke et al., the analysis of the temperaturedependent line width allows separation of the contributions of both terms. A plot of the Gaussian variance:

$$
w^{2}=2 \lambda_{\mathrm{CT}} k_{B} T+\sigma_{\mathrm{CT}}^{2}
$$

taken from Gaussian fits to the experimentally measured EL spectra [see Fig. 10(a)] for varying temperature should yield $2 \lambda_{\mathrm{CT}} k_{B}$ as the slope and the $y$ axis intersect is $\sigma_{\mathrm{CT}}^{2}$. From the data shown in Fig. 10(b) we see that $w^{2} / 2$ first decreases approximately linearly with a slope $\lambda_{\mathrm{CT}}=170$ $\mathrm{meV}$ (if the linear fit is forced to go through the origin). At $T<150 \mathrm{~K}$, however, it levels off and seems to saturate at about $w^{2}(T=0) / 2 k_{B}=25 \mathrm{eV} \mathrm{K}$, yielding $\sigma_{\mathrm{CT}}=65 \mathrm{meV}$. This value for the static disorder is in fairly good agreement with the inhomogenous line broadening obtained
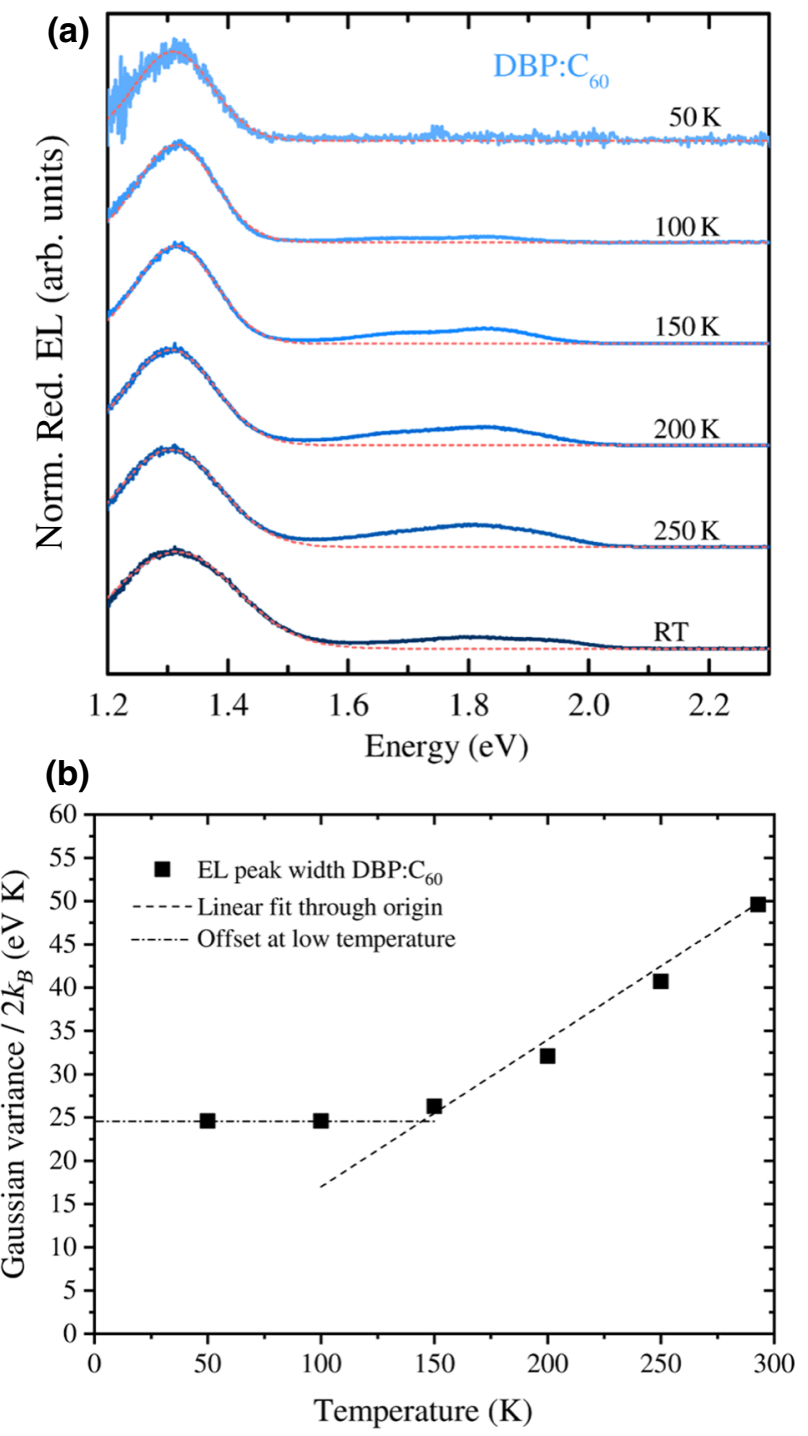

FIG. 10. (a) Temperature dependence of reduced EL spectra of DBP: $\mathrm{C}_{60}$ measured at a constant voltage of $2 \mathrm{~V}$. The peaks at about $1.3 \mathrm{eV}$ are fitted using simple Gaussian functions to determine their variance. (b) Gaussian variance of the EL peaks is plotted as a function of temperature to analyze the relative contributions of static and dynamic disorder according to Eq. (11).

from EL and IPCE spectra of single-donor devices. Nevertheless, it is apparent that the linear dependence predicted by Eq. (11) is not strictly fulfilled. It rather appears that there is temperature-independent offset determining the line width up to about $150 \mathrm{~K}$, before an increase (probably even stronger than linear with $T$ ) takes over. We want to note that the EL spectra are taken at constant voltage, implying that the carrier density is decreasing with lower temperature.

Turning again to the simulations on the temperature dependence of the EL spectra by the different models [see Figs. 8(c) and 8(d)], it is apparent that the Burke 
model predicts an EL peak shift towards lower energy with decreasing $T$, which is not observed. In the experiment the EL peak position of DBP: $\mathrm{C}_{60}$ is more or less independent of $T$ at about $1.31 \mathrm{eV}$ (see also Table SI-IV of the Supplemental Material [23]). In the Kahle model, in spite of the fact that it predicts a PL peak too high in energy, the line width exactly follows Eq. (11), which means that it yields a finite value at low $T$. The Marcus model, on the other hand, seems to work well at room temperature, but it predicts too strong line narrowing towards $0 \mathrm{~K}$. Thus, one has to conclude that none of the presented models works perfectly, because we observe contributions of both dynamic $\left(\lambda_{\mathrm{CT}}\right)$ and static disorder $\left(\sigma_{\mathrm{CT}}\right)$ being dominant in different temperature ranges.

Using Eq. (11) it is possible to set the Gaussian variance of the EL line shape caused only by static disorder in relation to the total variance by both static and dynamic disorder, which in turn allows their relative contributions at a given temperature to be quantified [51]:

$$
\begin{aligned}
D_{\text {stat }}(T) & =\frac{\sigma_{\mathrm{CT}}^{2}}{2 k_{B} T \lambda_{\mathrm{CT}}+\sigma_{\mathrm{CT}}^{2}} \quad \text { and } \\
D_{\text {dyn }} & =1-D_{\text {stat }} .
\end{aligned}
$$

For DBP at room temperature we obtain $D_{\text {stat }} \approx 33 \%$, meaning that static disorder is not the dominant contribution to the CT line width in agreement with the findings by Benduhn et al. [51] for a large number of donor:fullerene blends.

\section{CONCLUSIONS}

The central result of this work is summarized in Fig. 11. By combining photoelectron spectroscopy, probing the HOMO DOS in the ground state, with optical spectroscopy of the CT DOS in the excited state we obtain a comprehensive picture of the different energy landscapes in the two DA systems. In spite of their similar molecular structure and electronic properties the two donor molecules show largely different film morphology in blends with the fullerene $\mathrm{C}_{60}$, which leads to rather disparate spectroscopic behavior.

The crystalline donor DIP exhibits exponential tail states in both the HOMO as well as the CT DOS, which leads to pronounced state-filling effects, like a blueshift of the EL spectra with increasing carrier density. As a result, the analysis of CT absorption and emission spectra using Marcus theory (or any related model including static disorder) is questionable, because the DOS is non-Gaussian and the reciprocity relations are not strictly valid. As the main origin of tail states, we suggest grain boundaries between DIP domains with their particular "wedding-cake" terraces, where standing donor molecules expose their $\pi$-electron system to $\mathrm{C}_{60}$ acceptor molecules. Owing to the largescale phase separation this leads to a spatially nonuniform

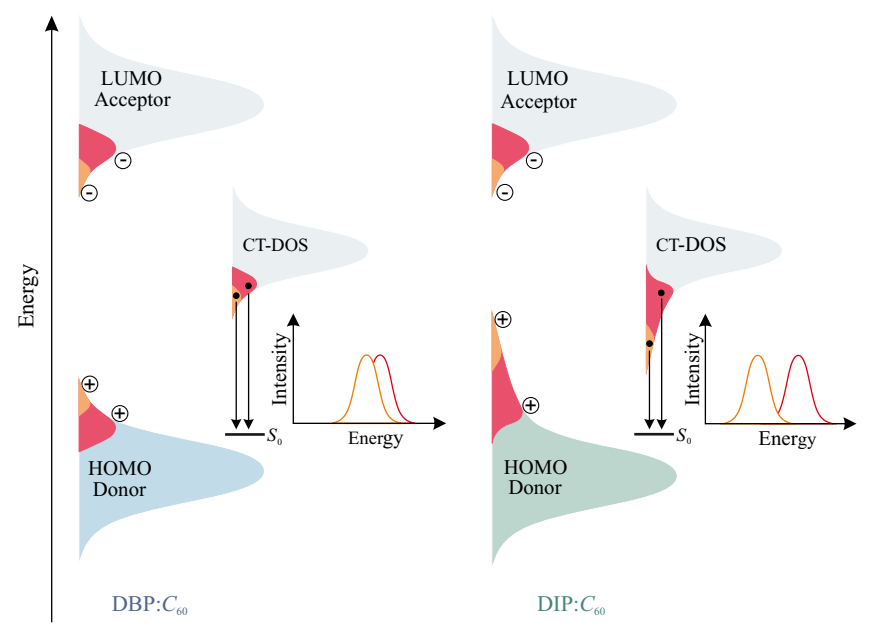

FIG. 11. Schematic illustration of the energy landscape at the two DA interfaces studied in this work. The occupation of interfacial CT states is indicated for low (orange) and high (red) charge-carrier density. For DBP: $\mathrm{C}_{60}$, shown on the left side, the CT DOS is Gaussian with little effect of state filling as evidenced by the almost negligible shift of the EL spectra. Whereas DIP: $\mathrm{C}_{60}$, shown on the right side, exhibits strong state filling effects with a concomitant shift of the EL peak upon increasing carrier density.

morphology with strong local variations in the electronic structure.

For the amorphous donor DBP, on the other hand, spatially homogenous, nonphase separating D:A blends are obtained that show very little indications for tail states in their DOS. Consequently, there is no significant spectral shift of their EL with increasing current and the CT spectra are well described by Marcus theory. At room temperature dynamic disorder caused by low-frequency modes of the DA molecules are the dominant line-broadening mechanism. Nevertheless, static disorder becomes relevant as well at low temperatures such that the line width does not completely narrow down upon cooling.

In summary, the perylene derivatives DIP and DBP are model systems to study different energy landscapes of CT states in DA solar cells resulting from their different blend morphology. Crystallinity and large-scale phase separation - in spite of the positive effect on charge transport - cause additional tail states and, thus, CT DOS broadening, which is expected to cause extra losses in the process of photogeneration of charge carriers.

\section{ACKNOWLEDGMENTS}

This work is funded by the German Research Foundation (DFG; Project No. BR 1728/19-1) and by the Bavarian State Ministry of Science, Research and the Arts within the collaborative research network "Solar Technologies go Hybrid (SolTech)." We thank the Japan Society for the Promotion of Science (JSPS) for supporting research stays 
at Chiba University within the JSPS Summer Program (T.L., A.H.) and a JSPS BRIDGE Fellowship (W.B.).

[1] M. A. Green, E. D. Dunlop, D. H. Levi, J. Hohl-Ebinger, M. Yoshita, and A. W. Y. Ho-Baillie, Solar cell efficiency tables (version 54), Prog. Photovoltaics: Res. Appl. 27, 565 (2019).

[2] R. A. J. Janssen and J. Nelson, Factors limiting device efficiency in organic photovoltaics, Adv. Mater. 25, 1847 (2013).

[3] T. Linderl, T. Zechel, M. Brendel, D. Moseguez, P. MüllerBuschbaum, J. Pflaum, and W. Brütting, Energy losses in small-molecule organic photovoltaics, Adv. Energy Mater. 7, 1700237 (2017).

[4] K. Vandewal, K. Tvingstedt, A. Gadisa, O. Inganäs, and J. V. Manca, Relating the open-circuit voltage to interface molecular properties of donor:acceptor bulk heterojunction solar cells, Phys. Rev. B 81, 125204 (2010).

[5] K. Vandewal, K. Tvingstedt, J. Manca, and O. Inganäs, Charge-transfer states and upper limit of the open-circuit voltage in polymer:fullerene organic solar cells, IEEE J. Sel. Top. Quantum Electron. 16, 1676 (2010).

[6] K. Vandewal, K. Tvingstedt, A. Gadisa, O. Inganäs, and J. V. Manca, On the origin of the open-circuit voltage of polymer-fullerene solar cells, Nat. Mater. 8, 904 (2009).

[7] A. Wilke, J. Endres, U. Hörmann, J. Niederhausen, R. Schlesinger, J. Frisch, P. Amsalem, J. Wagner, M. Gruber, A. Opitz, A. Vollmer, W. Brütting, A. Kahn, and N. Koch, Correlation between interface energetics and open circuit voltage in organic photovoltaic cells, Appl. Phys. Lett. 101, 233301 (2012).

[8] U. Hörmann, J. Kraus, M. Gruber, C. Schuhmair, T. Linderl, S. Grob, S. Kapfinger, K. Klein, M. Stutzman, H. J. Krenner, and W. Brütting, Quantification of energy losses in organic solar cells from temperature-dependent device characteristics, Phys. Rev. B 88, 235307 (2013).

[9] T. M. Burke, S. Sweetnam, K. Vandewal, and M. D. McGehee, Beyond langevin recombination: How equilibrium between free carriers and charge transfer states determines the open-circuit voltage of organic solar cells, Adv. Energy Mater. 5, 1500123 (2015).

[10] K. R. Graham, G. O. N. Ndjawa, S. M. Conron, R. Munir, K. Vandewal, J. J. Chen, S. Sweetnam, M. E. Thompson, A. Salleo, M. D. McGehee, and A. Amassian, The roles of structural order and intermolecular interactions in determining ionization energies and charge-transfer state energies in organic semiconductors, Adv. Energy Mater. 6, 1601211 (2016).

[11] X. Liu, K. Ding, A. Panda, and S. R. Forrest, Charge transfer states in dilute donor-acceptor blend organic heterojunctions, ACS Nano 10, 7619 (2016).

[12] G. O. N. Ndjawa, K. R. Graham, S. Mollinger, D. M. Wu, D. Hanifi, R. Prasanna, B. D. Rose, S. Dey, L. Yu, J.L. Brédas, M. D. McGehee, A. Salleo, and A. Amassian, Open-circuit voltage in organic solar cells: The impacts of donor semicrystallinity and coexistence of multiple interfacial charge-transfer bands, Adv. Energy Mater. 7, 1601995 (2017).
[13] U. Hörmann, C. Lorch, A. Hinderhofer, A. Gerlach, J. Gruber, M. Kraus, B. Sykora, S. Grob, T. Linderl, A. Wilke, A. Opitz, R. Hansson, A. S. Anselmo, Y. Ozawa, Y. Nakayama, H. Ishii, N. Koch, E. Moons, F. Schreiber, and W. Brütting, Voc from a morphology point of view: The influence of molecular orientation on the open circuit voltage of organic planar heterojunction solar cells, J. Phys. Chem. C 118, 26462 (2014).

[14] F.-J. Kahle, A. Rudnick, H. Bässler, and A. Köhler, How to interpret absorption and fluorescence spectra of charge transfer states in an organic solar cell, Mater. Horiz. 5, 837 (2018).

[15] Y. L. Lin, M. A. Fusella, and B. P. Rand, The impact of local morphology on organic donor/acceptor charge transfer states, Adv. Energy Mater. 8, 1702816 (2018).

[16] A. Melianas, N. Felekidis, Y. Puttisong, S. C. Meskers, O. Inganäs, W. M. Chen, and M. Kemerink, Nonequilibrium site distribution governs charge-transfer electroluminescence at disordered organic heterointerfaces, Proc. Natl. Acad. Sci. 116, 23416 (2019).

[17] J. Wagner, M. Gruber, A. Hinderhofer, A. Wilke, B. Bröker, J. Frisch, P. Amsalem, A. Vollmer, A. Opitz, N. Koch, F. Schreiber, and W. Brütting, High fill factor and open circuit voltage in organic photovoltaic cells with diindenoperylene as donor material, Adv. Funct. Mater. 20, 4295 (2010).

[18] M. Gruber, M. Rawolle, J. Wagner, D. Magerl, U. Hörmann, J. Perlich, S. V. Roth, A. Opitz, F. Schreiber, P. Müller-Buschbaum, and W. Brütting, Correlating structure and morphology to device performance of molecular organic donor-acceptor photovoltaic cells based on diindenoperylene (DIP) and $\mathrm{C}_{60}$, Adv. Energy Mater. 3, 1075 (2013).

[19] S. Grob, M. Gruber, A. N. Bartynski, U. Hörmann, T. Linderl, M. E. Thompson, and W. Brütting, Amorphous vs crystalline exciton blocking layers at the anode interface in planar and planar-mixed heterojunction organic solar cells, Appl. Phys. Lett. 104, 213304 (2014).

[20] M. Horlet, M. Kraus, W. Brütting, and A. Opitz, Diindenoperylene as ambipolar semiconductor: Influence of electrode materials and mobility asymmetry in organic field-effect transistors, Appl. Phys. Lett. 98, 233304 (2011).

[21] D. Fujishima, H. Kanno, T. Kinoshita, E. Maruyama, M. Tanaka, M. Shirakawa, and K. Shibata, Organic thin-film solar cell employing a novel electron-donor material, Solar Energy Mater. Solar Cells 93, 1029 (2009).

[22] A. Opitz, R. Banerjee, S. Grob, M. Gruber, A. Hinderhofer, U. Hörmann, J. Kraus, T. Linderl, C. Lorch, A. Steindamm, A. K. Topczak, A. Wilke, N. Koch, J. Pflaum, F. Schreiber, and W. Brütting, in Elementary Processes in Organic Photovoltaics, edited by K. Leo (Springer International, Basel, 2016), p. 77.

[23] See Supplemental Material at http://link.aps.org/supple mental/10.1103/PhysRevApplied.13.024061 for absorption spectra and characteristics of the samples in question. It also includes UPS, EL, PL, and EQE spectra. The Supplemental Material includes Refs. [8,14,18,19,30,45,52,53].

[24] A. Hinderhofer, A. Gerlach, K. Broch, T. Hosokai, K. Yonezawa, K. Kato, S. Kera, N. Ueno, and F. Schreiber, 
Geometric and electronic structure of templated $\mathrm{C}_{60}$ on diindenoperylene thin films, J. Phys. Chem. C 117, 1053 (2013).

[25] S. Grob, A. N. Bartynski, A. Opitz, M. Gruber, F. Grassl, E. Meister, T. Linderl, U. Hormann, C. Lorch, E. Moons, F. Schreiber, M. E. Thompson, and W. Brütting, Solvent vapor annealing on perylene-based organic solar cells, J. Mater. Chem. A 3, 15700 (2015).

[26] X. Xiao, J. D. Zimmerman, B. E. Lassiter, K. J. Bergemann, and S. R. Forrest, A hybrid planar-mixed tetraphenyldibenzoperiflanthene $/ \mathrm{C}_{7} 0$ photovoltaic cell, Appl. Phys. Lett. 102, 073302 (2013).

[27] S. Machida, Y. Ozawa, J. Takahashi, H. Tokairin, Y. Nakayama, and H. Ishii, Low-energy photoemission study of $\mathrm{C}_{60}$ / Rubrene/Au interfaces in practical device thickness, Appl. Phys. Express 6, 025801 (2013).

[28] T. Sato, K. R. Koswattage, Y. Nakayama, and H. Ishii, Density of states evaluation of an insulating polymer by highsensitivity ultraviolet photoemission spectroscopy, Appl. Phys. Lett. 110, 111102 (2017).

[29] T. Sato, H. Kinjo, J. Yamazaki, and H. Ishii, $10^{1} 5 \mathrm{~cm}^{-3} \mathrm{eV}^{-1}$ level detection of density of states of a p-type polymer by $h v$-dependent high-sensitivity ultraviolet photoemission spectroscopy, Appl. Phys. Express 10, 011602 (2017).

[30] Y. Nakayama, T. L. Nguyen, Y. Ozawa, S. Machida, T. Sato, H. Tokairin, Y. Noguchi, and H. Ishii, Complete demonstration of the valence electronic structure inside a practical organic solar cell probed by low energy photoemission, Adv. Energy Mater. 4, 1301354 (2014).

[31] S. Kera, H. Yamane, and N. Ueno, First-principles measurements of charge mobility in organic semiconductors: Valence hole-vibration coupling in organic ultrathin films, Prog. Surf. Sci. 84, 135 (2009).

[32] M. Schwarze, C. Gaul, R. Scholz, F. Bussolotti, A. Hofacker, K. S. Schellhammer, B. Nell, B. D. Naab, Z. Bao, D. Spoltore, K. Vandewal, J. Widmer, S. Kera, N. Ueno, F. Ortmann, and K. Leo, Molecular parameters responsible for thermally activated transport in doped organic semiconductors, Nat. Mater. 18, 242 (2019).

[33] H. Nicolai, M. Kuik, G. Wetzelaer, B. de Boer, C. Campbell, C. Risko, J. Brédas, and P. Blom, Unification of trap-limited electron transport in semiconducting polymers, Nat. Mater. 11, 882 (2012).

[34] G. Zuo, M. Linares, T. Upreti, and M. Kemerink, General rule for the energy of water-induced traps in organic semiconductors, Nat. Mater. 18, 588 (2019).

[35] A. K. Topczak, T. Roller, B. Engels, W. Brütting, and J. Pflaum, Nonthermally activated exciton transport in crystalline organic semiconductor thin films, Phys. Rev. B 89, 201203 (2014).

[36] N. S. Sariciftci, Role of buckminsterfullerene $\mathrm{C}_{60}$ in organic photoelectric devices, Prog. Quantum Electron. 19, 131 (1995).

[37] M. Fox, Optical Properties of Solids (Oxford University Press, Oxford, 2010).

[38] V. Capozzi, G. Casamassima, G. Lorusso, A. Minafra, R. Piccolo, T. Trovato, and A. Valentini, Optical spectra and photoluminescence of $\mathrm{C}_{60}$ thin films, Solid State Commun. 98, 853 (1996).

[39] M. Heilig, M. Domhan, and H. Port, Optical properties and morphology of thin diindenoperylene films, J. Lumin. 110, 290 (2004).

[40] D. Zhang, A. Horneber, J. Mihaljevic, U. Heinemeyer, K. Braun, F. Schreiber, R. Scholz, and A. Meixner, Plasmon resonance modulated photoluminescence and raman spectroscopy of diindenoperylene organic semiconductor thin film, J. Lumin. 131, 502 (2011).

[41] P. Würfel and U. Würfel, Physics of Solar Cells - From Basic Principles to Advanced Concepts, (Wiley-VCH, Berlin, 2016).

[42] K. Vandewal, J. Benduhn, and V. C. Nikolis, How to determine optical gaps and voltage losses in organic photovoltaic materials, Sustainable Energy Fuels 2, 538 (2018).

[43] U. Heinemeyer, R. Scholz, L. Gisslén, M. I. Alonso, J. O. Ossó, M. Garriga, A. Hinderhofer, M. Kytka, S. Kowarik, A. Gerlach, and F. Schreiber, Exciton-phonon coupling in diindenoperylene thin films, Phys. Rev. B 78, 085210 (2008).

[44] L. Ferlauto, F. Liscio, E. Orgiu, N. Masciocchi, A. Guagliardi, F. Biscarini, P. Samorì, and S. Milita, Enhancing the charge transport in solution-processed perylene di-imide transistors via thermal annealing of metastable disordered films, Adv. Funct. Mater. 24, 5503 (2014).

[45] A. Köhler and H. Bässler, Electronic Processes in Organic Semiconductors: An Introduction (Wiley-VCH, Weinheim, 2015).

[46] N. A. Ran, J. A. Love, C. J. Takacs, A. Sadhanala, J. K. Beavers, S. D. Collins, Y. Huang, M. Wang, R. H. Friend, G. C. Bazan, and T.-Q. Nguyen, Harvesting the full potential of photons with organic solar cells, Adv. Mater. 28, 1482 (2016).

[47] J. Benduhn, K. Tvingstedt, F. Piersimoni, S. Ullbrich, Y. Fan, M. Tropiano, K. A. McGarry, O. Zeika, M. K. Riede, C. J. Douglas, S. Barlow, S. R. Marder, D. Neher, D. Spoltore, and K. Vandewal, Intrinsic non-radiative voltage losses in fullerene-based organic solar cells, Nat. Energy 2, 17053 (2017).

[48] C. Faber, J. L. Janssen, M. Côté, E. Runge, and X. Blase, Electron-phonon coupling in the $\mathrm{C}_{60}$ fullerene within the many-body GW approach, Phys. Rev. B 84, 155104 (2011).

[49] W. Gong, M. A. Faist, N. J. Ekins-Daukes, Z. Xu, D. D. Bradley, J. Nelson, and T. Kirchartz, Influence of energetic disorder on electroluminescence emission in polymer: Fullerene solar cells, Phys. Rev. B 86, 024201 (2012).

[50] S. Roland, J. Kniepert, J. A. Love, V. Negi, F. Liu, P. Bobbert, A. Melianas, M. Kemerink, A. Hofacker, and D. Neher, Equilibrated charge carrier populations govern steady-state nongeminate recombination in disordered organic solar cells, J. Phys. Chem. Lett. 10, 1374 (2019).

[51] J. Benduhn, Ph.D. thesis, TU Dresden, 2019.

[52] S. Grob, Ph.D. thesis, University of Augsburg, 2015.

[53] B. R. Sykora, Master's thesis, University of Augsburg and Universidade de Aveiro, 2013. 